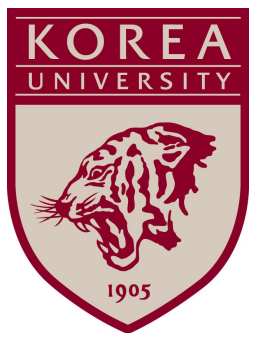

Discussion Paper Series

No. 1007

March 2010

\title{
What is an oil shock? Panel data evidence
}

\author{
Dong Heon Kim
}

The Institute of Economic Research - Korea University

Anam-dong, Sungbuk-ku, Seoul, 136-701, South Korea, Tel: (82-2) 3290-1632, Fax: (82-2) 928-4948

Copyright (C) 2010 IER. 


\title{
What is an oil shock? \\ Panel data evidence
}

\author{
Dong Heon Kim* \\ Department of Economics \\ Korea University
}

Revised: March 17, 2010

\begin{abstract}
This paper characterizes the nonlinear relation between oil price change and GDP growth, focusing on the panel data of various industrialized countries. Toward this end, the paper extends a flexible nonlinear inference to the panel data analysis where the random error components are incorporated into the flexible approach. The paper reports clear evidence of nonlinearity in the panel and confirms earlier claims in the literature - oil price increases are much more important than decreases and previous upheaval in oil prices causes the marginal effect of any given oil price change to be reduced. Our result suggests that the nonlinear oil-macroeconomy relation is generally observable over different industrialized countries and it is desirable for one to use the nonlinear function of oil price change for GDP forecast.
\end{abstract}

JEL Classification: E32; C33

Keywords: Oil shock; Nonlinear flexible inference; Panel data; Error components model, Economic fluctuation

\footnotetext{
0* Associate Professor, Department of Economics, Korea University, 5-1 Anam-dong, Seongbuk-Gu, Seoul, 136-701, South Korea, Tel: +82 23290 2226, Fax: +82 2928 4948, Email: dongkim@korea.ac.kr.
} 


\section{Introduction}

Quite a few studies have reported that changes in the price of oil appear to have a significant effect on economic activity. Examples include Rasche and Tatom (1977, 1981), Hamilton (1983,1996, 2003, 2008, 2009a), Burbidge and Harrison (1984), Gisser and Goodwin (1986), Mork (1989), Dotsey and Reid (1992), Lee, Ni, and Ratti (1995), Rotemberg and Woodford (1996), Carruth, Hooker and Oswald (1998), Davis and Haltiwanger (2001), Cuñado and Pérez de Gracia (2003), Lee and Ni (2002), Leduc and Sill (2004), Hamilton and Herrera (2004), among others. However, Hooker (1996) argues that since the mid-1980s, the linear relation between oil prices and economic activity appears to be either unstable or misspecified. Blanchard and Galí (2008) show that the effects of a given change in the price of oil have changed substantially over time and conclude that three hypotheses - i) more flexible labor market over time, ii) changes in the way of conducting monetary policy, and iii) a decline in the share of oil in the economyseem to have played an important role in explaining the different effects of oil prices during the 1970s and during the last decade.

In the recent studies about the oil-macroeconomy relation, particularly two issues were debated. The first issue is what causes the oil price increases. Hamilton (1996, 2003, 2009a) claims that physical disruptions of supply of oil caused significant impact of oil price changes on macroeconomy whereas Barsky and Kilian $(2002,2004)$ and Kilian (2008a, 2008b, 2009a) argue that expectations of future oil supply interruptions induce shocks to the precautionary demand for oil that reflect fears about future oil supplies while exogenous oil supply shocks account for only a comparatively small part of oil price movement and thus the oil price increases were driven by strong global demand for oil in conjunction with capacity constraints in crude oil production. ${ }^{1}$ The second issue is the functional form of the oil-macroeconomy relation. Lee et al. (1995),

\footnotetext{
${ }^{1}$ Hamilton (2009a) claims that the price run-up of 2007-2008 was caused by strong demand confronting stagnating world production whereas previous oil price shocks were primarily caused by physical disruptions of supply but the consequences of the 2007-2008 oil price increases for the economy appear to have been similar to those observed in earlier episodes about the U.S. recessions caused oil price shocks.
} 
Hamilton (1996, 2003, 2009a), Cuñado and Pérez de Gracia (2003), and Jiménez-Rodriguez and Sánchez (2005) show that the relation is nonlinear while Kilian and Vigfusson (2009) find little evidence on the nonlinear relation.

This paper focuses on the latter issue. Many authors have concluded that the nonlinearity of the relation between oil prices and economic activity is responsible for the instability of the empirical relation or misspecification of the functional form. ${ }^{2}$ Hamilton (2003) applies a flexible approach to nonlinear inference developed by Hamilton (2001) and tries to isolate an exogenous component of oil price movements by measuring the oil supply curtailed by five separate military conflicts during the postwar period to address a statistically significant nonlinear relation. Hamilton (2003) finds that the nonlinear relation of oil prices suggested by the functional form of the conditional expectation function supports the lines suggested in the literature: oil price increases are much more important than oil price decreases and increases have significantly less predictive content if they simply correct previous decreases. He also finds that the nonlinear transformation of oil prices based on the functional form is in fact quite similar to the first-stage least-squares fit from a regression of oil price changes on these exogenous supply disturbances, and that the dynamic multipliers from the nonlinear relation are similar to those coming from a linear relation estimated by instrumental variables. Mork, Olsen, and Mysen (1994) conclude from the study of seven OECD countries that the correlation patterns between oil-price change and real GDP growth are not the same for price increases and decreases, and asymmetry in the effects of oil price fluctuation is a reasonably robust empirical finding. Cuñado and Pérez de Gracia (2003) found from the use of different transformation of oil price data that similar evidence of nonlinearity is observed for European countries as well as U.S.A. while there are significant differences among some of the countries. Jiménez-Rodriguez and Sánchez (2005) carried out multivariate VAR analysis using both linear and non-linear models for main industrialized countries and found that oil price increases have an impact on GDP growth of a larger magnitude than that of oil price declines, supporting a non-linear impact of oil prices on real GDP.

\footnotetext{
${ }^{2}$ Examples include Loungani (1986), Davis (1987a, 1987b), Mork (1989), Lee et al. (1995), Hamilton (1996, 2003), Davis and Haltiwanger (2001), Cuñado and Pérez de Gracia (2003), and Jiménez-Rodriguez and Sánchez (2005), among others.
} 
Kilian and Vigfusson (2009), however, show that the regression models and estimation methods which use measures that censor energy price changes to exclude all energy price decreases and are typically used in the existing literature, produce inconsistent estimates of the true effects of unanticipated energy prices increases and lead to overestimating the impact of energy price shocks on macroeconomic aggregates. They find little evidence against the null hypothesis of symmetry in the responses to energy price shocks. Nevertheless, Hamilton (2009b) notes that it must be differences in the specification and data set between Hamilton (2003) and Kilian and Vigfusson (2009), rather than differences in the testing methodology, that accounts for the different findings, and provides a number of detail differences that could explain less possible rejection of the null hypothesis of linearity obtained by Kilian and Vigfusson (2009) such as different data sets, different measure of oil prices, different price adjustment, the inclusion of contemporaneous regressors, and number of lags.

This paper considers two insights to examine the functional form of the oil-macroeconomy relationship. First of all, some studies suggest that the nonlinear relationship between oil price changes and real GDP growth is present not only within a cross-section unit (a country) but also over the cross-section units (multi-countries). To the best of my knowledge, however, there is no systematic investigation of whether the relation between oil price change and real GDP is nonlinear in terms of panel data analysis. This paper extends Hamilton's (2001) methodology to the panel framework to characterize the nonlinear relation. Specifically, we consider the error components model of Wallace and Hussain (1969), Baltagi, Song, and Jung (2002), among others, in the context of a flexible approach to nonlinear inference of Hamilton (2001). ${ }^{3}$ The methodology developed in this paper is useful for analyzing nonlinear relation between economic variables in the panel framework because the benefits of nonlinear flexible inferences claimed in Hamilton (2001) can be strengthened with several advantages from using panel data. ${ }^{4}$ This

\footnotetext{
${ }^{3}$ The error component model has been considered by Wallace and Hussain (1969), Nerlove (1971), Maddala (1971), Amemiya (1971), Swamy and Arora (1972), Baltagi (1981), Baltagi and Griffin (1983), Boehmer and Megginson (1990), and Baltagi and Pinnoi (1995), among others. For further discussion and references see Baltagi (2008).

${ }^{4}$ For detail discussion of the benefits from using panel data, see Klevmarken (1989), Hsiao (2003), and Baltagi (2008).
} 
framework may be applied to re-examine the structural stability of the Phillips Curve as the example of Hamilton (2001) and investigate the nature of nonlinearity in the monetary policy rule as the case of Kim, Osborn and Sensier (2005) in the panel data. Secondly, our parametric approach does not have to use the censored oil price changes to investigate asymmetric oilmacroeconomy relation and thus one would avoid potential problems from using the censored energy price changes as pointed out in Kilian and Vigfusson (2009).

In our model, the nonlinear functional relation is common across countries and over time and the regression error is assumed to be composed of three independent components - one component associated with the cross sectional units, another with an aggregate shock, and the third being an idiosyncratic shock. The results support the claim of a nonlinear relation along the lines suggested in the literature: oil price increases affect the economy whereas decreases do not and previous upheaval in oil prices causes a reduction in the marginal effect of any given oil price change. The alternative specifications of the panel data model with nonlinear flexible inference as a robustness analysis confirm the asymmetric effect of oil price change on macroeconomy.

The plan of the paper is as follows: Section 2 considers the error components model of the panel data in the context of a parametric approach to flexible nonlinear inference. Empirical results for the analysis of oil-macroeconomy relation are presented in Section 3. Concluding remarks are offered in Section 4.

\section{A parametric approach to nonlinear flexible inference in the panel}

\subsection{Model}

Consider the general nonlinear regression model of the form based on the panel

$$
y_{i t}=\mu_{i}\left(\mathbf{x}_{i t}\right)+\varepsilon_{i t}, i=1,2, \ldots, N, t=1,2, \ldots, T,
$$


where, $y_{i t}$ is a scalar dependent variable at time $t$ for country $i, \mathbf{x}_{i t}^{\prime}$ are $k$-dimensional vector of explanatory variables, and $\varepsilon_{i t}$ is i.i.d. with mean zero and independent of both $\mu_{i}($.$) and \mathbf{x}_{i \tau}$ for $i=1, \ldots, N$, and $\tau=t, t-1, \ldots, 1$. This specification considers the nonlinear relation over the group as well as within the group and thus allows the functional relation to be different over cross-country units. Following Hamilton (2001), the conditional mean function in the panel data, $\mu_{i}\left(\mathbf{x}_{i t}\right)$, is written as

$$
\mu_{i}\left(\mathbf{x}_{i t}\right)=\alpha_{0}+\boldsymbol{\alpha}_{i 1}^{\prime} \mathbf{x}_{i t}+\lambda_{i} m\left(\mathbf{g}_{i} \odot \mathbf{x}_{i t}\right), i=1,2, \ldots, N
$$

where $m($.$) is denotes the realization of a scalar-valued Gaussian random field with mean$ zero and unit variance, $\alpha_{0}, \boldsymbol{\alpha}_{i 1}^{\prime}, \lambda_{i}$, and $\mathbf{g}_{i}$ are population parameters to be estimated, $\mathbf{g}_{i}=$ $\left(g_{i 1}, g_{i 2}, \ldots, g_{i k}\right)^{\prime}$ and $\odot$ indicates element-by-element multiplication. $\lambda_{i}^{2}$ governs the overall importance of the nonlinear component, and $\mathbf{g}_{i}$ governs the variability of the nonlinear component with respect to each explanatory variable.

As an approach to combining cross section and time series data, we consider the use of an error components model where one component of random error $\varepsilon_{i t}$ is an unobserved individual effect which is constant through time, another component is an unobserved time effect which is the same for all individuals at a given time, and the third component is an unobserved remainder which differs among individuals both at a point in time and through time. Thus, we assume that the residual, $\varepsilon_{i t}$, is decomposed into the sum of three components:

$$
\varepsilon_{i t}=\omega_{i}+a_{t}+v_{i t},
$$

where, $\omega_{i}$ is an individual specific variable, $a_{t}$ is a time-specific variable, and $v_{i t}$ is the remainder. $\omega_{i}^{\prime} s, a_{t}^{\prime} s$, and $v_{i t}^{\prime} s$ are random, have zero means, have variances $\sigma_{\omega}^{2}, \sigma_{a}^{2}$, and $\sigma_{v}^{2}$, and are independent of each other. That is, it is assumed that $E \omega_{i}=E a_{t}=E v_{i t}=0, E \omega_{i} \omega_{j}=0$ for $i \neq j, E a_{t} a_{s}=0$ for $t \neq s, E v_{i t} v_{j s}=\sigma_{v}^{2}$ for $i=j, t=s$, and zero otherwise, $E \omega_{i} a_{t}=E \omega_{i} v_{i t}=E a_{t} v_{i t}=0$. In addition, $\mathbf{x}_{i t}$ is independent of $\omega_{i}, a_{t}$, and $v_{i t}$ for all $i$ and $t$, assuming that the regressor $\mathbf{x}_{i t}$ is strictly exogenous and $\mathbf{x}_{i t}$ and $\varepsilon_{i t}$ are independent of 
the realization of the random field $m(\cdot)$ in equation (2).

For the case of strictly exogenous regressors and no lagged dependent variables, we assume that conditional on the full sample of observations on the exogenous explanatory variables $(X=$ $\left.\left\{\mathbf{x}_{i t}\right\}_{i=1, \ldots, N ; t=1, \ldots, T}\right)$, the variables $\omega_{i}, a_{t}$ and $v_{i t}$ are all Normal with zero means, variances $\sigma_{\omega}^{2}$, $\sigma_{a}^{2}$, and $\sigma_{v}^{2}$, respectively, and are mutually independent. That is, for $\varepsilon=\left(\varepsilon_{1}^{\prime}, \ldots, \boldsymbol{\varepsilon}_{N}^{\prime}\right)^{\prime}$ and $\varepsilon_{i}=\left(\varepsilon_{i 1}, \ldots, \varepsilon_{i T}\right)^{\prime}$, we assume that

$$
\varepsilon \mid X \sim N\left(\mathbf{0}, \sigma_{\omega}^{2}\left(\mathbf{I}_{N} \otimes \mathbf{J}_{T}\right)+\sigma_{a}^{2}\left(\mathbf{J}_{N} \otimes \mathbf{I}_{T}\right)+\sigma_{v}^{2} \mathbf{I}_{N T}\right)
$$

where $\mathbf{J}_{T}$ and $\mathbf{J}_{N}$ denote $(T \times T)$ and $(N \times N)$ matrices of ones, respectively. In our application below we include lagged values of $y_{i t}$ along with lagged values of oil prices, with the latter taken to be strictly exogenous. In this case the conditioning set $X$ corresponds to the full sample of observations on oil prices and the pre-sample observations on $y_{i t}$, that is, $X=\left\{\left\{o_{i t}\right\}_{i=1, \ldots, N ; t=-3,-2, \ldots, T},\left\{y_{i t}\right\}_{i=1, . ., N ; t=-3,-2,-1,0}\right\}$ for the above conditional distribution.

For simplicity, we further assume that the slopes in the linear component in equation (2) are homogenous among different individuals, and $\lambda_{i}$ and $\mathbf{g}_{i}$ are not specific to cross-section units. In general, to allow nonlinear parameters to be country-specific (heterogenous nonlinear components), may be useful for considering the panel heterogeneity issue in the application of our method to various economic application. In our application to the industrialized countries for oil-macroeconomy relation, however, such heterogeneity across the panel may be less likely and the homogenous assumption for nonlinear parameters over different countries would make one focus on common oil-macroeconomy relation across countries.

Under these assumptions, the general specification, (1) and (2), with random-effect and $k$ explanatory variables and the conditional mean function of equation (2) in the panel can be rewritten 


$$
\begin{aligned}
y_{i t} & =\alpha_{0}+\boldsymbol{\alpha}_{1}^{\prime} \mathbf{x}_{i t}+\lambda m\left(\mathbf{g} \odot \mathbf{x}_{i t}\right)+\varepsilon_{i t}, \\
\varepsilon_{i t} & =\omega_{i}+a_{t}+v_{i t}, \\
\mu\left(\mathbf{x}_{i t}\right) & =\alpha_{0}+\boldsymbol{\alpha}_{1}^{\prime} \mathbf{x}_{i t}+\lambda m\left(\mathbf{g} \odot \mathbf{x}_{i t}\right), i=1,2, \ldots, N, t=1, \ldots, T .
\end{aligned}
$$

Hamilton (2001) chooses a generalized version of the so-called spherical covariance function and this function can be applied to the panel as

$$
\begin{aligned}
H_{k}\left(h_{i s, j t}\right) & = \begin{cases}G_{k-1}\left(h_{i s, j t}, 1\right) / G_{k-1}(0,1) & \text { if } h_{i s, j t} \leq 1 \\
0 & \text { if } h_{i s, j t}>1,\end{cases} \\
G_{k}\left(h_{i s, j t}, r\right) & =\int_{h_{i s, j t}}^{r}\left(r^{2}-z^{2}\right)^{k / 2} d z, \\
h_{i s, j t} & =(1 / 2)\left\{\left[\mathbf{g} \odot\left(\mathbf{x}_{i s}-\mathbf{x}_{j t}\right)\right]^{\prime}\left[\mathbf{g} \odot\left(\mathbf{x}_{i s}-\mathbf{x}_{j t}\right)\right]\right\}^{1 / 2}, i, j=1, . ., N, t, s=1, . ., T
\end{aligned}
$$

where $H_{k}\left(h_{i s, j t}\right)$ denotes the $\{i s, j t\}$ entry in the $N T \times N T$ covariance function matrix $H_{k}$.

\subsection{Inference about the conditional expectation function}

If each component in $\varepsilon_{i t}$ (equation (5)) is random and normally distributed and if the regressor $\mathbf{x}_{i t}^{\prime}$ is strictly exogenous, then the specification of equations (4), (5) and (6) implies a GLS regression model of the form

$$
\mathbf{y} \mid \mathbf{X} \sim N(\mathbf{X} \boldsymbol{\beta}, \boldsymbol{\Omega})
$$


where

$$
\begin{aligned}
\mathbf{y}= & \left(\mathbf{y}_{1}^{\prime}, \ldots, \mathbf{y}_{N}^{\prime}\right)^{\prime}, \mathbf{y}_{i}=\left(y_{i 1}, \ldots, y_{i T}\right)^{\prime}, \\
\mathbf{X}= & {\left[\begin{array}{cc}
1 & \mathbf{x}_{11}^{\prime} \\
1 & \mathbf{x}_{12}^{\prime} \\
\cdot & \cdot \\
1 & \mathbf{x}_{1 T}^{\prime} \\
\cdot & \cdot \\
1 & \mathbf{x}_{N T}^{\prime}
\end{array}\right] } \\
\mathbf{\Omega} & =\mathbf{C}_{0}+\sigma_{\omega}^{2}\left(\mathbf{I}_{N} \otimes \mathbf{J}_{T}\right)+\sigma_{a}^{2}\left(\mathbf{J}_{N} \otimes \mathbf{I}_{T}\right)+\sigma_{v}^{2} \mathbf{I}_{N T}, \\
\mathbf{C}_{0}= & {\left[\lambda^{2} H_{k}\left(h_{i s, j t}\right)\right]_{i, j=1, . ., N, \& t, s=1, \ldots, T}, }
\end{aligned}
$$

$\boldsymbol{\beta}=\left(\alpha_{0}, \boldsymbol{\alpha}_{1}^{\prime}\right)^{\prime}$, the $(1+k)$-dimensional vector, $\mathbf{J}_{T}$ and $\mathbf{J}_{N}$ are the $T \times T$ and the $N \times N$ ones matrixes respectively, $\mathbf{I}_{N T}$ is the $N T \times N T$ identity matrix, the function $H_{k}($.$) is as specified in$ equations (4) and (5), and $h_{i s, j t}$ is given in equation (9). Note that $\sigma^{2}=\sigma_{\omega}^{2}+\sigma_{a}^{2}+\sigma_{v}^{2}$.

The log likelihood function is

$$
\begin{aligned}
& \ln f\left(\mathbf{y} ; \boldsymbol{\beta}, \sigma_{\omega}^{2}, \sigma_{a}^{2}, \sigma_{v}^{2}, \lambda, \mathbf{g}\right) \\
= & -\frac{N T}{2} \ln 2 \pi-\frac{1}{2} \ln |\boldsymbol{\Omega}|-\frac{1}{2}(\mathbf{y}-\mathbf{X} \boldsymbol{\beta})^{\prime} \boldsymbol{\Omega}^{-1}(\mathbf{y}-\mathbf{X} \boldsymbol{\beta}) .
\end{aligned}
$$

The maximum likelihood estimators of $\boldsymbol{\beta}, \sigma_{\omega}^{2}, \sigma_{a}^{2}, \sigma_{v}^{2}, \lambda$, and $\mathbf{g}$ are obtained by maximizing the log likelihood function (13) with respect to those parameters. However, even if the $\boldsymbol{\varepsilon}=\mathbf{y}-\mathbf{X} \boldsymbol{\beta}$, were observable, it is very difficult to solve explicitly this maximization problem. Hamilton (2001) shows that one useful way to deal with this problem is to use reparameterization to obtain the concentrated likelihood function. For each pair of observation $i s$ and $j t$, calculate $\widetilde{\mathbf{x}}_{i t}=$ $\mathbf{g} \odot \mathbf{x}_{i t}$ and $h_{i s, j t}(\mathbf{g})=(1 / 2)\left[\left(\widetilde{\mathbf{x}}_{i s}-\widetilde{\mathbf{x}}_{j t}\right)^{\prime}\left(\widetilde{\mathbf{x}}_{i s}-\widetilde{\mathbf{x}}_{j t}\right)\right]^{1 / 2}$. Let $\mathbf{H}(\mathbf{g})$ denote the $(N T \times N T)$ matrix whose $(i s, j t)$ element is $H_{k}\left(h_{i s, j t}(\mathbf{g})\right)$. Let $\mathbf{Y}_{n t}=\left(\mathbf{y}_{n t}^{\prime}, \mathbf{x}_{n t}^{\prime}, \mathbf{y}_{n t-1}^{\prime}, \mathbf{x}_{n t-1}^{\prime}, \ldots, \mathbf{y}_{n 1}^{\prime}, \mathbf{x}_{n 1}^{\prime}\right)^{\prime}, \mathbf{y}_{n t}=$ $\left(y_{1 t}, y_{2 t}, \ldots, y_{n t}\right)^{\prime}$ and $\mathbf{x}_{n t}=\left(\mathbf{x}_{1 t}^{\prime}, \mathbf{x}_{2 t}^{\prime}, \ldots, \mathbf{x}_{n t}^{\prime}\right)^{\prime}$, denote information observed through date $t$ for $n$ 
number of individuals. Define $\zeta \equiv \lambda / \sigma_{v}$ to be the ratio of the standard deviation of the nonlinear component $\lambda m(\mathbf{x})$ to that of the residual $v$ and let $\phi_{\omega}=\frac{\sigma_{\omega}}{\sigma_{v}}, \phi_{a}=\frac{\sigma_{a}}{\sigma_{v}} \boldsymbol{\psi}=\left(\alpha_{0}, \boldsymbol{\alpha}^{\prime}, \sigma_{v}^{2}\right)^{\prime}, \boldsymbol{\theta}=$ $\left(\mathbf{g}^{\prime}, \zeta, \phi_{\omega}, \phi_{a}\right)^{\prime}$ and

$$
\mathbf{W}(\mathbf{X} ; \boldsymbol{\theta}) \equiv \zeta^{2} \mathbf{H}(\mathbf{g})+\phi_{\omega}^{2}\left(\mathbf{I}_{N} \otimes \mathbf{J}_{T}\right)+\phi_{a}^{2}\left(\mathbf{J}_{N} \otimes \mathbf{I}_{T}\right)+\mathbf{I}_{N T}
$$

Now the log likelihood can be written from (13)

$$
\begin{aligned}
& \ln f(\mathbf{y} ; \boldsymbol{\psi}, \boldsymbol{\theta}) \\
= & -\frac{N T}{2} \ln 2 \pi-\frac{N T}{2} \ln \sigma_{v}^{2}-\frac{1}{2} \ln |\mathbf{W}(\mathbf{X} ; \boldsymbol{\theta})| \\
& -\frac{1}{2 \sigma_{v}^{2}}(\mathbf{y}-\mathbf{X} \boldsymbol{\beta})^{\prime} \mathbf{W}(\mathbf{X} ; \boldsymbol{\theta})^{-1}(\mathbf{y}-\mathbf{X} \boldsymbol{\beta}) .
\end{aligned}
$$

For given $\boldsymbol{\theta}$, the value of $\boldsymbol{\psi}$ that maximizes (15) can be calculated analytically as

$$
\begin{aligned}
\widetilde{\boldsymbol{\beta}}(\boldsymbol{\theta}) & =\left[\mathbf{X}^{\prime} \mathbf{W}(\mathbf{X} ; \boldsymbol{\theta})^{-1} \mathbf{X}\right]^{-1}\left[\mathbf{X}^{\prime} \mathbf{W}(\mathbf{X} ; \boldsymbol{\theta})^{-1} \mathbf{y}\right] \\
\tilde{\sigma}_{v}^{2} & =(\mathbf{y}-\mathbf{X} \boldsymbol{\beta})^{\prime} \mathbf{W}(\mathbf{X} ; \boldsymbol{\theta})^{-1}(\mathbf{y}-\mathbf{X} \boldsymbol{\beta}) / N T .
\end{aligned}
$$

Now, these allow us to concentrate the log likelihood (15) as

$$
\begin{aligned}
L_{c}(\boldsymbol{\theta} ; \mathbf{y}, \mathbf{X}) & =\sum_{i=1}^{N} \sum_{t=1}^{T} \ln f\left(y_{i t} \mid \mathbf{x}_{i t}, \mathbf{Y}_{N t-1} ; \widetilde{\boldsymbol{\psi}}(\boldsymbol{\theta}), \boldsymbol{\theta}\right) \\
& =-\frac{N T}{2} \ln 2 \pi-\frac{N T}{2} \ln \widetilde{\sigma}_{v}^{2}(\boldsymbol{\theta})-\frac{1}{2} \ln |\mathbf{W}(\mathbf{X} ; \boldsymbol{\theta})|-(N T / 2) .
\end{aligned}
$$

Numerically maximizing (18) gives the MLE $\widehat{\boldsymbol{\theta}}$, which from (16) and (17) gives $\widehat{\boldsymbol{\psi}}$. 


\subsection{Bayesian Analysis}

In this subsection, we extend Hamilton's (2001) Bayesian analysis to the error components model of the panel data considered above. Let $\boldsymbol{\psi}=\left(\boldsymbol{\beta}^{\prime}, \sigma_{v}^{-2}\right)^{\prime}$, and $\boldsymbol{\theta}=\left(\mathbf{g}^{\prime}, \zeta, \phi_{\omega}, \phi_{a}\right)^{\prime}$. We adopt a standard prior for the linear components as in Hamilton $(2001,2003) .^{5}$ The prior distribution of $\sigma_{v}^{-2}$ is $\Gamma(v, \xi)$ :

$$
p\left(\sigma_{v}^{-2}\right)=\frac{\xi^{\nu}}{\Gamma(\nu)} \sigma_{v}^{-2(\nu-1)} \exp \left[-\xi \sigma_{v}^{-2}\right]
$$

where $\nu=0.25$ and $\xi=\left(\nu s_{y}^{2} / 2\right), s_{y}^{2}=\frac{1}{N T} \sum_{i=1}^{N} \sum_{t=1}^{T}\left(y_{i t}-\bar{y}\right)^{2}, \bar{y}=\frac{1}{N T} \sum_{i=1}^{N} \sum_{t=1}^{T} y_{i t}$. The prior distribution of $\boldsymbol{\beta}$ conditional on $\sigma_{v}^{-2}$ is Gaussian:

$$
p\left(\boldsymbol{\beta} \mid \sigma_{v}^{-2}\right)=\frac{1}{\left(2 \pi \sigma_{v}^{2}\right)^{(k+1) / 2}}|\mathbf{M}|^{-1 / 2} \times \exp \left[\left(\frac{-1}{2 \sigma_{v}^{2}}\right)(\boldsymbol{\beta}-\mathbf{m}) \mathbf{M}^{-1}(\boldsymbol{\beta}-\mathbf{m})\right],
$$

where we set the first element of $\mathbf{m}$ to the sample mean of $y_{i t}$ and all other components to zero, $\mathbf{m}=(\bar{y}, 0, \ldots, 0)^{\prime}$ and we take $\mathbf{M}=N T\left(\mathbf{X}^{\prime} \mathbf{X}\right)^{-1}$, so that the prior has the weight of a single observation on $\left(y_{i t}, \mathbf{x}_{i t}^{\prime}\right)$. The prior distribution of each element of $\boldsymbol{\theta}$ is a lognormal distribution:

$$
p(\boldsymbol{\theta})=\prod_{i=1}^{k+3} \frac{1}{\sqrt{2 \pi} \tau_{i} \theta_{i}} \exp \left[\frac{-\left[\ln \left(\theta_{i}\right)-\vartheta_{i}\right]^{2}}{2 \tau_{i}^{2}}\right] .
$$

Note that the prior for $\theta_{i}$ is taken to be independent of that for $\boldsymbol{\beta}, \sigma_{v}^{-2}$ and $\theta_{j}, j \neq i$. We use $\tau_{i}=1$ for $i=1, . ., k+3$, and allow $\vartheta_{j}$ to depend on the standard deviation of variable $j, \vartheta_{j}=-\ln \left(\sqrt{k s_{j}^{2}}\right)$ with $s_{j}^{2}=\frac{1}{N T} \sum_{i=1}^{N} \sum_{t=1}^{T}\left(x_{j i t}-\bar{x}_{j}\right)^{2}$ and $\vartheta_{\omega}=-\ln \left(\sqrt{\frac{s_{\omega}^{2}}{s_{y}^{2}}}\right), \vartheta_{a}=-\ln \left(\sqrt{\frac{s_{a}^{2}}{s_{y}^{2}}}\right)$, where $s_{\omega}^{2}=N^{-1} \sum_{i=1}^{N}\left(\bar{y}_{i .}-\bar{y}\right)^{2}, s_{a}^{2}=T^{-1} \sum_{t=1}^{T}\left(\bar{y}_{. t}-\bar{y}\right)^{2}, \bar{y}_{i .}=T^{-1} \sum_{t=1}^{T} y_{i t}, \bar{y}_{. t}=N^{-1} \sum_{i=1}^{N} y_{i t}$. $\vartheta_{k+1}=0$.

Our interest is to infer the posterior expected value of some function $l(\boldsymbol{\theta})$,

$$
E\left[l(\boldsymbol{\theta}) \mid \mathbf{Y}_{N T}\right]=\int l(\boldsymbol{\theta}) f\left(\boldsymbol{\theta} \mid \mathbf{Y}_{N T}\right) d \boldsymbol{\theta}
$$

\footnotetext{
${ }^{5}$ As in Hamilton $(2001,2003)$ for time-series analysis, the method here requires nondiffuse priors in order for the posterior distribution to be well-defined. For further details about the prior distribution, see Hamilton (2001).
} 
where $f\left(\boldsymbol{\theta} \mid \mathbf{Y}_{N T}\right)=f(\boldsymbol{\theta}, \mathbf{y} \mid \mathbf{X}) / \int f(\boldsymbol{\theta}, \mathbf{y} \mid \mathbf{X}) d \boldsymbol{\theta}$. Following Geweke (1989) and Hamilton (2001), we evaluate (22) by using importance-sampling algorithm. We consider an arbitrary importance sampling distribution $I(\boldsymbol{\theta})$ and generate an artificial i.i.d. sample $\boldsymbol{\theta}^{(1)}, \boldsymbol{\theta}^{(2)}, \ldots, \boldsymbol{\theta}^{(D)}$ drawn from the $I(\boldsymbol{\theta})$, and calculate

$$
\begin{gathered}
\hat{E}\left[l(\boldsymbol{\theta}) \mid \mathbf{Y}_{N T}\right]=l(\boldsymbol{\theta})^{*}=\frac{\sum_{j=1}^{D} l\left(\boldsymbol{\theta}^{(j)}\right) w\left(\boldsymbol{\theta}^{(j)}, \mathbf{Y}_{N T}\right)}{\sum_{j=1}^{D} w\left(\boldsymbol{\theta}^{(j)}, \mathbf{Y}_{N T}\right)} \\
w\left(\boldsymbol{\theta}^{(j)}, \mathbf{Y}_{N T}\right)=\frac{f\left(\boldsymbol{\theta}^{(j)}, \mathbf{y} \mid \mathbf{X}\right)}{I\left(\boldsymbol{\theta}^{(j)}\right)}
\end{gathered}
$$

Following Hamilton (2001), we have an algorithm based on a truncated mixture density. With probability 0.5 , we generate $\boldsymbol{\theta}$ from a multivariate Student $t$ distribution with $\varphi=2$ degrees of freedom, centered at the MLE with precision matrix given by $(-1 / 2)$ times the matrix of second derivatives of the log likelihood function. With probability 0.5 , the elements of $\ln (\boldsymbol{\theta})$ are drawn independently from $N\left(\vartheta_{i}, 4\right)$ distributions, so that the logs have the same mean but twice the standard deviation of the prior. After we threw out any draw for which some $\theta_{i}<0$, the truncation was achieved. Thus, we have

$$
\begin{aligned}
I(\boldsymbol{\theta}) \propto & (0.5) \frac{\Gamma[(k+3+\varphi) / 2]}{\Gamma(\varphi / 2)(\varphi \pi)^{(k+3) / 2}}|\hat{\boldsymbol{\Omega}}|^{-1 / 2} \times\left[1+\varphi^{-1}(\boldsymbol{\theta}-\hat{\boldsymbol{\theta}})^{\prime} \hat{\boldsymbol{\Omega}}^{-1}(\boldsymbol{\theta}-\hat{\boldsymbol{\theta}})\right]^{-1(k+3+\varphi) / 2} \\
& +(0.5) \prod_{j=1}^{k+3} \frac{1}{\sqrt{2 \pi}\left(2 \tau_{j}\right) \theta_{j}} \exp \left[\frac{-\left[\ln \left(\theta_{j}\right)-\vartheta_{j}\right]^{2}}{2\left(2 \tau_{j}\right)^{2}}\right], \\
\text { for } \theta_{j} \geq & 0, j=1,2, \ldots, k+3,
\end{aligned}
$$

where the constant of proportionality reflects the truncation, $\hat{\boldsymbol{\theta}}$ is the MLE, $\hat{\boldsymbol{\Omega}}$ is twice its asymptotic variance, $\varphi=2, \tau_{j}=1, \vartheta_{j}=0$, for $j=k+1, k+2, k+3$ and $\vartheta_{j}$ is given by in the part of the prior distribution $\vartheta_{j}=-\ln \left(\sqrt{k s_{j}^{2}}\right)$ for $j=1, \ldots, k$. 


\subsection{Testing for nonlinearity}

We consider Hamilton's (2001) LM test in the error components model of the panel data. In what follows, we briefly describe the test procedure. Following Hamilton (2001) we fix $\mathbf{g}$ from the scale of the data, for example, by setting $g_{i}$ equal to the mean of the prior distribution in (21). Let $\boldsymbol{\sigma}^{2}=\left(\sigma_{\omega}^{2}, \sigma_{a}^{2}, \sigma_{v}^{2}\right)^{\prime}$ and suppose these variances are observable. Let $\mathbf{H}_{N T}$ be a known $(N T \times N T)$ positive semidefinite matrix and let

$$
\boldsymbol{\Omega}_{N T}=\lambda^{2} \mathbf{H}_{N T}+\sigma_{\omega}^{2} \mathbf{Q}_{\omega}+\sigma_{a}^{2} \mathbf{Q}_{a}+\sigma_{v}^{2} \mathbf{I}_{N T}
$$

where $\mathbf{Q}_{\omega}=\left(\mathbf{I}_{N} \otimes \mathbf{J}_{T}\right), \mathbf{Q}_{a}=\mathbf{J}_{N} \otimes \mathbf{I}_{T}$. Consider the likelihood function under the assumption that $\mathbf{y} \mid \mathbf{X}, \boldsymbol{\sigma}^{\mathbf{2}} \sim N\left(\mathbf{X} \boldsymbol{\beta}, \boldsymbol{\Omega}_{N T}\right)$ :

$$
\ln f\left(\mathbf{y} \mid \mathbf{X}, \boldsymbol{\sigma}^{\mathbf{2}} ; \boldsymbol{\zeta}\right)=-\frac{N T}{2} \ln 2 \pi-\frac{1}{2} \ln \left|\boldsymbol{\Omega}_{N T}\right|-\frac{1}{2} \operatorname{tr}\left(\boldsymbol{\Omega}_{N T}{ }^{-1} \varepsilon \varepsilon^{\prime}\right),
$$

for $\boldsymbol{\varepsilon}=\mathbf{y}-\mathbf{X} \boldsymbol{\beta}$ and $\boldsymbol{\zeta}=\left(\lambda^{2}, \boldsymbol{\beta}^{\prime}\right)^{\prime}$. Then, we have a score:

$$
\left.\frac{\partial \ln f\left(\mathbf{y} \mid \mathbf{X}, \boldsymbol{\sigma}^{\mathbf{2}} ; \boldsymbol{\zeta}\right)}{\partial \lambda^{2}}\right|_{\lambda^{2}=0}=-(1 / 2) \operatorname{tr}\left(\mathbf{Q}_{N T}^{-1} \mathbf{H}_{N T}\right)+(1 / 2) \operatorname{tr}\left(\mathbf{Q}_{N T}^{-1} \mathbf{H}_{N T} \mathbf{Q}_{N T}^{-1} \boldsymbol{\varepsilon} \varepsilon^{\prime}\right]
$$

where $\mathbf{Q}_{N T}=\sigma_{\omega}^{2} \mathbf{Q}_{\omega}+\sigma_{a}^{2} \mathbf{Q}_{a}+\sigma_{v}^{2} \mathbf{I}_{N T}$. The information matrix is:

$$
-E\left\{\left.\frac{\partial^{2} \ln f\left(\mathbf{y} \mid \mathbf{X}, \boldsymbol{\sigma}^{2} ; \boldsymbol{\zeta}\right)}{\partial \boldsymbol{\zeta} \partial \boldsymbol{\zeta}^{\prime}}\right|_{\boldsymbol{\zeta}=\boldsymbol{\zeta}_{0}}\right\}=\left[\begin{array}{cc}
(1 / 2) \operatorname{tr}\left[\left(\mathbf{Q}_{N T}^{-1} \mathbf{H}_{N T}\right)^{2}\right] & \mathbf{0} \\
\mathbf{0} & \sigma^{-2} \mathbf{X}^{\prime} \mathbf{X}
\end{array}\right]
$$

Then, the Lagrange multiplier test of the null hypothesis that $\lambda=0$ conditional on $\sigma^{2}$ is given by:

$$
\aleph_{N T}=\frac{\left[\left(\varepsilon^{\prime} \mathbf{Q}_{N T}^{-1} \mathbf{H}_{N T} \mathbf{Q}_{N T}^{-1} \varepsilon\right)-t r\left(\mathbf{Q}_{N T}^{-1} \mathbf{H}_{N T}\right)\right]}{\sqrt{\operatorname{tr}\left[\left(\mathbf{Q}_{N T}^{-1} \mathbf{H}_{N T}\right)^{2}\right]}} .
$$

Following Wallace and Hussain (1969) and Amemiya (1971) for the best quadratic unbi- 
ased estimators of the variance components, we turn to estimates of $\varepsilon_{i t}$, say $\hat{\varepsilon}_{i t}$ which are observed residuals obtained by least squares. Amemiya (1971) points out that the estimate of variance components based on the ordinary least squares are less efficient and provides following process: $\varepsilon$ is obtained by first estimating $\boldsymbol{\beta}$ by $\hat{\boldsymbol{\beta}}=\left(\mathbf{X}^{\prime} \mathbf{G X}\right)^{-1} \mathbf{X}^{\prime} \mathbf{G y}, \mathbf{G}=\mathbf{I}_{N T}-$ $(1 / T) \mathbf{Q}_{\omega}-(1 / N) \mathbf{Q}_{a}+(1 / N T) \mathbf{J}_{N T}, \mathbf{J}_{N T}$ is the $N T \times N T$ matrix consisting only of ones, and $\alpha_{0}$ by $\hat{\alpha}_{0}=(1 / N T) \mathbf{e}_{N T}^{\prime}(\mathbf{y}-\mathbf{X} \hat{\boldsymbol{\beta}})$ and then predicting $\boldsymbol{\varepsilon}$ by $\mathbf{y}-\hat{\alpha}_{0} \mathbf{e}_{N T}-\mathbf{X} \hat{\boldsymbol{\beta}}=\mathbf{y}-\left(\mathbf{J}_{N T} / N T\right) \mathbf{y}$ $-(\mathbf{1} / N T) \mathbf{J}_{N T} \mathbf{X}\left(\mathbf{X}^{\prime} \mathbf{G X}\right)^{-1} \mathbf{X}^{\prime} \mathbf{G y}-\mathbf{X}\left(\mathbf{X}^{\prime} \mathbf{G X}\right)^{-1} \mathbf{X}^{\prime} \mathbf{G y}$. Then, the analysis of variance estimates are

$$
\begin{aligned}
\hat{\sigma}_{v}^{2} & =\frac{1}{(N-1)(T-1)} \sum_{i=1}^{N} \sum_{t=1}^{T}\left(\hat{\varepsilon}_{i t}-\frac{1}{T} \hat{\varepsilon}_{i .}-\frac{1}{N} \hat{\varepsilon}_{. t}+\frac{1}{N T} \hat{\varepsilon}_{. .}\right)^{2} \\
& =\frac{1}{(N-1)(T-1)} \hat{\varepsilon}^{\prime} \mathbf{G} \hat{\varepsilon}, \\
\hat{\sigma}_{\omega}^{2} & =\frac{1}{T(N-1)} \sum_{i=1}^{N}\left(\frac{1}{T} \hat{\varepsilon}_{i .}-\frac{1}{N T} \hat{\varepsilon}_{. .}\right)^{2}-\frac{1}{T} \hat{\sigma}_{v}^{2} \\
& =\frac{1}{T(N-1)(T-1)} \hat{\varepsilon}^{\prime}\left[\frac{T-1}{T} \mathbf{Q}_{\omega}-\frac{T-1}{N T} \mathbf{J}_{N T}-\mathbf{G}\right] \hat{\varepsilon}, \\
\hat{\sigma}_{a}^{2} & =\frac{1}{N(T-1)} \sum_{t=1}^{T}\left(\frac{1}{N} \hat{\varepsilon}_{. t}-\frac{1}{N T} \hat{\varepsilon}_{. .}\right)^{2}-\frac{1}{N} \hat{\sigma}_{v}^{2} \\
& =\frac{1}{N(N-1)(T-1)} \hat{\varepsilon}^{\prime}\left[\frac{N-1}{N} \mathbf{Q}_{a}-\frac{N-1}{N T} \mathbf{J}_{N T}-\mathbf{G}\right] \hat{\varepsilon},
\end{aligned}
$$

where $\hat{\varepsilon}_{i .}=\sum_{t=1}^{T} \hat{\varepsilon}_{i t}, \hat{\varepsilon}_{. t}=\sum_{i=1}^{N} \hat{\varepsilon}_{i t}, \hat{\varepsilon}_{. .}=\sum_{i=1}^{N} \sum_{t=1}^{T} \hat{\varepsilon}_{i t}$. The Lagrange multiplier test of (30) with the estimate of variance components (31), (32) and (33) is given

$$
\hat{\aleph}_{N T}=\frac{\left[\left(\hat{\varepsilon}^{\prime} \hat{\mathbf{Q}}_{N T}^{-1} \mathbf{H}_{N T} \hat{\mathbf{Q}}_{N T}^{-1} \hat{\varepsilon}\right)-\operatorname{tr}\left(\hat{\mathbf{Q}}_{N T}^{-1} \mathbf{H}_{N T}\right)\right]}{\sqrt{\operatorname{tr}\left[\left(\hat{\mathbf{Q}}_{N T}^{-1} \mathbf{H}_{N T}\right)^{2}\right]}},
$$

where $\hat{\boldsymbol{\varepsilon}}=\mathbf{y}-\hat{\alpha}_{0} \mathbf{e}_{N T}-\mathbf{X} \hat{\boldsymbol{\beta}}, \hat{\alpha}_{0}=(1 / N T) \mathbf{e}_{N T}^{\prime}(\mathbf{y}-\mathbf{X} \hat{\boldsymbol{\beta}}), \hat{\boldsymbol{\beta}}=\left(\mathbf{X}^{\prime} \mathbf{G X}\right)^{-1} \mathbf{X}^{\prime} \mathbf{G y}$, and $\hat{\mathbf{Q}}_{N T}=$ $\hat{\sigma}_{\omega}^{2} \mathbf{Q}_{\omega}+\hat{\sigma}_{a}^{2} \mathbf{Q}_{a}+\hat{\sigma}_{v}^{2} \mathbf{I}_{N T}$. 


\section{Empirical results}

\subsection{Data}

The countries included in this study are Australia, Canada, Germany, Netherlands, U.K., and U.S.A. ${ }^{6}$ The series used for real output, $y_{i t}$ is the quarterly growth rate of real GDP and we use the world produce price index for crude oil for all countries in dollars and convert it into each country's currency by means of the market exchange rate. The sample used is from 1960:I to 2003:IV. All these data have been downloaded from the International Financial Statistics (IFS) in IMF except U.S.A. The data for U.S. real GDP are from the Bureau of Economic Analysis (BEA) and the produce price index for crude oil for U.S.A. are from the Bureau of Labor Statistics. A total of $T \times N=1026$ observations is available.

\subsection{Linear error components model}

When $\lambda=0$, the model of equations (4), (5) and (6) is a two-way error component model as follows:

$$
\begin{aligned}
y_{i t} & =\alpha_{0}+\boldsymbol{\alpha}_{1}^{\prime} \mathbf{x}_{i t}+\varepsilon_{i t}, \\
\varepsilon_{i t} & =\omega_{i}+a_{t}+v_{i t}, i=1,2, \ldots, N, t=1, \ldots, T .
\end{aligned}
$$

Following Amemiya (1971), we consider the interactive MLE for equations (35) and (36) and the estimation results are as follows:

\footnotetext{
${ }^{6}$ We initially considered 10 countries (G7 + Australia, Netherlands, Sweden) and excluded four countries, French, Italy, Japan and Sweden. The reason was that as the result of the estimation of the linear model for individual country, these countries have shown quite different dynamics over lagged GDP growth and lagged oil price change, implying that the slopes in the linear component in equation (2.2) are not homogenous among different individuals and thus indicating that these countries are far away from homogenous assumption. Japan, in particular, exhibits signficantly different dynamics over lagged GDP growth during the sample. An anomymous referee notes that this empirical section may have potential issues arising due to the limited country numbers. This issue would be serious if we estimated the unrestricted model (1)-(2) with small samples. However, it is unlikely that the estimation of the restricted model (4)-(6) representing a behavioral equation with the same parameters over time and across countries raises the issue.
} 


$$
\begin{aligned}
& y_{i t}=\underset{(0.282)}{2.531}-\underset{(0.032)}{0.044} y_{i t-1}+\underset{(0.031)}{0.068} y_{i t-2}+\underset{(0.031)}{0.123} y_{i t-3}+\underset{(0.031)}{0.068} y_{i t-4} \\
& +\underset{(0.014)}{0.011} o_{i t-1}-\underset{(0.015)}{0.005 o_{i t-2}}-\underset{(0.014)}{0.023 o_{i t-3}}-\underset{(0.014)}{0.039} o_{i t-4} \\
& \hat{\sigma}_{\omega}^{2}=0.035, \hat{\sigma}_{a}^{2}=2.750, \hat{\sigma}_{v}^{2}=21.39 \text {. }
\end{aligned}
$$

The coefficient on $o_{i t-4}$ is statistically significant at the $1 \%$ level. Even though the results seem to support the linear relationship between oil price change and real GDP growth, the test statistic of the null hypothesis of linearity has a value of 30.93 , which for a $\chi^{2}(1)$ variable implies overwhelming rejection of the null hypothesis that the relation is linear in the panel. There seems little question that the relation between oil prices and GDP is nonlinear.

To investigate the performance of the test statistics (34), instead of deriving the asymptotic approximation in equation (34), we try to approximate the exact small-sample distribution of $\hat{\aleph}_{N T}$ by Monte Carlo methods. Given the data generating process of the linear error components model, we calculated the test statistic $\hat{\aleph}_{N T}$ for 1,000 simulations and did not find any case where $\hat{\aleph}_{N T}$ is greater than that of our original data. The highest $\hat{\aleph}_{N T}$ among 1000 simulations was $4.46\left(\chi^{2}(1)\right.$ form of the test $\left.=19.89\right)$ while the $\hat{\aleph}_{N T}$ of the original data was $5.56\left(\chi^{2}(1)\right.$ form of the test $=30.93)$, implying that the original data for the relation between oil price and GDP growth is far from the linear relation. Furthermore, at the nominal 5\% level of significance, the rejection rate of the linearity null hypothesis was $1.6 \%$, implying that overall performance of the developed test statistic (34) has good small-sample property. The Appendix provides detail Monte Carlo methods for the small-sample distribution of the LM test statistic of equation (34).

\subsection{Nonlinear flexible model with random effect error components}

Bayesian posterior estimates and their standard errors for the flexible nonlinear alternative with error components as in the model of equations (4), (5) and (6) are as follows: 


$$
\begin{aligned}
& y_{i t}=\underset{(0.530)}{2.114}-\underset{(0.033)}{0.056} y_{i t-1}+\underset{(0.028)}{0.050} y_{i t-2}+\underset{(0.033)}{0.109} y_{i t-3}+\underset{(0.030)}{0.056} y_{i t-4} \\
& +\underset{(0.014)}{0.010 o_{i t-1}}-\underset{(0.015)}{0.003 o_{i t-2}}-\underset{(0.015)}{0.020 o_{i t-3}}-\underset{(0.015)}{0.038 o_{i t-4}} \\
& \left.\left.+\underset{(0.118)}{4.567[0.054)} \underset{(0.336 m}{0.080 o_{i t-1}} \underset{(0.08)}{0.07 o_{i t-2}}, \underset{(0.08)}{0.07 o_{i t-3}} \underset{(0.07)}{0.07 o_{i t-4}}\right)+\tilde{\omega}_{i}+\tilde{a}_{t}+\tilde{v}_{i t}\right] \\
& \hat{\sigma}_{\omega}^{2}=0.982, \hat{\sigma}_{a}^{2}=0.476, \hat{\sigma}_{v}^{2}=20.86
\end{aligned}
$$

where $\tilde{\omega}_{i} \sim N(0,1), \tilde{a}_{t} \sim N(0,1), \tilde{v}_{i t} \sim N(0,1)$, and $m($.$) denotes an unobserved realization$ from a Gaussian random field with mean zero, unit variance and correlations given by equations (7)-(9). ${ }^{7}$ The innovation $\omega_{i}, a_{t}$, and $v_{i t}$ in (5) are written as $\sigma_{v}=4.567$ times $\tilde{\omega}_{i}, \sigma_{v}$ times $\tilde{a}_{t}$ and $\sigma_{v}$ times $\tilde{v}_{i t}$ respectively. The parameter $\lambda$ in (4) is written as $\sigma_{v}$ times the parameter $\zeta$, whose estimate is 0.336 . Each of the four lags of oil price changes exert an overall negative effect on output growth as indicated by the linear coefficients, though only the coefficient on $o_{i t-4}$ is statistically significant. Although one would accept a hypothesis of linearity for any one of the lags of oil prices taken individually (as reflected by the insignificant $t$-statistics on the individual coefficients $g_{i}$ ), collectively the nonlinear component makes a highly significant contribution (as evidenced by the $t$-statistic for $\zeta=0$ or the LM tests).

To take a look at what the nonlinear function $\mu($.$) looks like and compare the nonlinear$ function of Hamilton (2003) with that of the panel data, I performed an exercise similar to Hamilton (2003) and fixed the values of $o_{i t-2}, o_{i t-3}$, and $o_{i t-4}$ equal to their sample means and examined the consequences of changing $o_{i t-1}$ alone, that is, I set $\mathbf{x}^{*}=\left(x_{i 1}, \bar{o}, \bar{o}, \bar{o}\right)$ and evaluated the Bayesian posterior expectation of the optimal inference of the value of the unobserved function $\mu\left(\mathbf{x}^{*}\right)$. Figure 1 plots the result as a function of $x_{i 1}$ along with $95 \%$ probability regions. The implied function is nonlinear, suggesting that if oil prices either increase or decrease after three quarters of stability, slightly slower GDP growth is predicted than if oil prices had remained stable, though decreases are a little worse news than increases. Nevertheless, the confidence band

\footnotetext{
${ }^{7}$ The Bayesian analysis is based on 100,000 draws from the importance sampling density described in Subsection $(2.3)$.
} 
of the Figure 1 indicates that the change in GDP growth does not appear to be statistically significant.

Figure 2 and Figure 3 answer the analogous question, fixing $o_{i t-1}, o_{i t-3}$, and $o_{i t-4}$ at their sample means and varying the value of $o_{i t-2}$ in Figure 2 and fixing $o_{i t-1}, o_{i t-2}$, and $o_{i t-4}$ at their sample means and varying the value of $o_{i t-3}$ in Figure 3. Both Figure 2 and Figure 3 show different impression from that of Figure 1 though the implied function is nonlinear. Both figures indicate the opposite situation to the Figure 1 where oil price increases are worse news than decreases. However, in the Figure 2 and the Figure 3, the confidence interval is quite broad, implying that such an inference might not be warranted statistically.

Figure 4 describes the effect of $o_{i t-4}$ alone and shows more dramatic relation, suggesting that decreases in oil prices four quarters earlier have almost no consequences for current GDP growth, whereas oil price increases significantly reduce expected GDP growth. Furthermore, the confidence interval shows a statistically significant relation. This figure indicates an asymmetric specification as in Mork's (1989) and Hamilton (1996, 2003).

I calculated how $o_{i t-3}$ is affected by different values of $o_{i t-4}$ to examine the interactive effects. Figure 5 compares the three functions $\hat{\mu}\left(\bar{o}, \bar{o}, x_{i 3}, 0\right), \hat{\mu}\left(\bar{o}, \bar{o}, x_{i 3}, 5\right), \hat{\mu}\left(\bar{o}, \bar{o}, x_{i 3},-5\right)$, plotted as a function of $x_{i 3}$. The dotted line represents the relation between various $x_{i 3}$ and $o_{i t-4}=0$, which is essentially the same as the mean value plotted in Figure 3. The solid line represents the relation to show how the effect of an $x_{i 3} \%$ oil price change three quarters ago would be different if oil prices had also increased $5 \%$ the quarter before that. The one point to make is that the solid line is uniformly lower than the dotted line. This implies that regardless of the value of $o_{i t-3}$, one forecasts definitely lower GDP growth when an oil price increase four quarters earlier than when an unchanged oil price four quarters earlier. Furthermore, one can notice that the slope of the dotted line is steeper than that of the solid line, implying that the additional information content of any change in quarter $t-3$ is reduced by an oil price increase four quarters earlier. The dashed line plots the predicted GDP growth for quarter $t$ when $o_{i t-3}=x_{i 3}$ and $o_{i t-4}=-5$. The almost same shape of the dotted line and the dashed line indicates that if oil prices decreased four quarters earlier, this has little consequences for forecasting GDP if it was followed by no 
change in oil price in quarter $t-3$. In other words, one should downweight another oil price change in period $t-3$ following a $5 \%$ decrease in period $t-4$. The overall conclusion of Figure 5 supports the view of Lee et al. (1995) and Hamilton $(1996,2003)$ that previous upheaval in oil prices tends to reduce the marginal effect of any given oil price change.

In sum, our results of the panel data analysis confirm the findings of Hamilton (2003, 2009b), Cuñado and Pérez de Gracia (2003), and Jiménez-Rodriguez and Sánchez (2005). The relation between oil price change and GDP growth is nonlinear and oil price increases are much more important than oil price decreases and increases that come after a long period of stable prices have a bigger effect than those that simply correct previous decreases for industrialized countries as well as U.S.A.

\subsection{Alternative specifications}

To examine whether the results of previous section rely on the specification of flexible nonlinear error component model we consider other two alternative specifications. The first alternative is a flexible nonlinear model with fixed effects instead of random error components and this specification is as follows:

$$
\begin{aligned}
y_{i t} & =\alpha_{i}+\boldsymbol{\alpha}_{1}^{\prime} \mathbf{x}_{i t}+\lambda m\left(\mathbf{g} \odot \mathbf{x}_{i t}\right)+\varepsilon_{i t}, \\
\varepsilon_{i t} & =a_{t}+v_{i t}, \\
\mu\left(\mathbf{x}_{i t}\right) & =\alpha_{i}+\boldsymbol{\alpha}_{1}^{\prime} \mathbf{x}_{i t}+\lambda m\left(\mathbf{g} \odot \mathbf{x}_{i t}\right), i=1,2, \ldots, N, t=1, \ldots, T,
\end{aligned}
$$

where $\alpha_{i}$ denotes the individual specific effect and it is assumed that $\alpha_{i}$ is a fixed parameter to be estimated, $a_{t} \sim$ i.i.d.N $\left(0, \sigma_{a}^{2}\right)$ and $v_{i t} \sim i . i . d . N\left(0, \sigma_{v}^{2}\right)$. Inference in this case is conditional on the particular $N$ individuals. The other alternative is a flexible nonlinear model without the individual specific effect and we have a following specification: 


$$
\begin{aligned}
y_{i t} & =\alpha_{0}+\boldsymbol{\alpha}_{1}^{\prime} \mathbf{x}_{i t}+\lambda m\left(\mathbf{g} \odot \mathbf{x}_{i t}\right)+\varepsilon_{i t}, \\
\varepsilon_{i t} & =a_{t}+v_{i t}, \\
\mu\left(\mathbf{x}_{i t}\right) & =\alpha_{0}+\boldsymbol{\alpha}_{1}^{\prime} \mathbf{x}_{i t}+\lambda m\left(\mathbf{g} \odot \mathbf{x}_{i t}\right), i=1,2, \ldots, N, t=1, \ldots, T .
\end{aligned}
$$

Inference in this case is for the case of pooling across countries but not for the case of pooling over time.

The test statistic of the null hypothesis of linearity for the case of linear fixed-effect model and for the case of linear pooling model without the country specific effect has a value of 28.41 and 26.99 respectively, which are similar with the case of linear random error component model and both values for a $\chi^{2}(1)$ variable indicate strong rejection of the null hypothesis of linearity. Following the reparametrization as in Subsection 2.2, we have Bayesian posterior estimates and their standard errors for the model (40)-(41) and the model (43)-(44) as follows:

$$
\begin{aligned}
& y_{i t}=\alpha_{i}-\underset{(0.033)}{0.055} y_{i t-1}+\underset{(0.030)}{0.050} y_{i t-2}+\underset{(0.032)}{0.105} y_{i t-3}+\underset{(0.033)}{0.049} y_{i t-4} \\
& +\underset{(0.014)}{0.016 o_{i t-1}}-\underset{(0.014)}{0.002 o_{i t-2}}-\underset{(0.016)}{0.023 o_{i t-3}}-\underset{(0.016)}{0.037 o_{i t-4}} \\
& \left.+\underset{(0.122)}{4.826[(0.056)}\left[\underset{(0.09)}{0.294 m} \underset{(0.07}{0.07 t-1}, \underset{(0.08)}{0.07} o_{i t-2}, \underset{(0.07)}{0.06} o_{i t-3}, \underset{(0.07)}{0.06 o_{i t-4}}\right)+\tilde{a}_{t}+\tilde{v}_{i t}\right] \\
& \hat{\sigma}_{a}^{2}=0.886, \hat{\sigma}_{v}^{2}=23.29,
\end{aligned}
$$

$$
\begin{aligned}
& y_{i t}=\underset{(0.463)}{2.081}-\underset{(0.032)}{0.053} y_{i t-1}+\underset{(0.030)}{0.060 y_{i t-2}}+\underset{(0.033)}{0.107} y_{i t-3}+\underset{(0.030)}{0.059} y_{i t-4} \\
& +\underset{(0.014)}{0.010 o_{i t-1}}-\underset{(0.014)}{0.003 o_{i t-2}}-\underset{(0.014)}{0.023 o_{i t-3}}-\underset{(0.015)}{0.036 o_{i t-4}} \\
& \left.+\underset{(0.111)}{4.587[(0.054)}\left[\underset{(0.10)}{0.336 m} \underset{(0.09}{0.09} o_{i t-1}, \underset{(0.10)}{0.08} o_{i t-2}, \underset{(0.08)}{0.07} o_{i t-3}, \underset{(0.07)}{0.07} o_{i t-4}\right)+\tilde{a}_{t}+\tilde{v}_{i t}\right] \\
& \hat{\sigma}_{a}^{2}=0.964, \hat{\sigma}_{v}^{2}=21.04,
\end{aligned}
$$


where $\alpha_{i}$ is a fixed parameter to be estimated for the country specific effect. ${ }^{8}$ The values of estimated fixed parameter $\alpha_{i}$ in the nonlinear fixed effect model (45) are between 1.49 and 2.58 over all sample countries and all estimated fixed parameters are statistically significant.

Estimated parameter governing the overall importance of the nonlinear component $\lambda$, is written as $\zeta \cdot \sigma_{v}$ and their estimates in both models are statistically significant and are quite similar to those of the nonlinear random effect model in equation (38). Furthermore, in both models, the coefficient on $o_{i t-4}$ is statistically significant at the $5 \%$ level and the value of the coefficient is close to that of equation (38). Figure $6 \mathrm{a}$ and $6 \mathrm{~b}$ describe the effect of $o_{i t-4}$ alone on current economic growth based on the nonlinear fixed effect model (45) and on the nonlinear pooling model (46) respectively. Interestingly, both figures are quite similar with Figure 4, suggesting that two alternative specifications supports similar nonlinear oil-macroeconomy relation which the nonlinear random error component model of equation (38) showed. Figure 7a and $7 \mathrm{~b}$ plot the effect of oil price on GDP growth three quarter later for different possible values of $o_{i t-4}-\hat{\mu}\left(\bar{o}, \bar{o}, x_{i 3}, 0\right), \hat{\mu}\left(\bar{o}, \bar{o}, x_{i 3}, 5\right)$, and $\hat{\mu}\left(\bar{o}, \bar{o}, x_{i 3},-5\right)$ - to examine the view that previous turbulence in oil prices causes the marginal effect of any given oil price change to be reduced. Both figures are also quite similar with Figure 5 in the case of nonlinear random error component model of equation (38). Overall, two alternative specifications supports similar oil-macreconomy relation as in the nonlinear random error component model and thus the claim of nonlinear relation seems to be robust to the nonlinear flexible inferenence with various specifications of the panel data model.

\section{Concluding remarks}

The instability over time in a linear regression of output growth on lagged oil prices has triggered the investigation of functional relation between oil price and real economic activity. Hamilton (2003) shows by employing the methodology of nonlinear flexible inference of Hamilton (2001) that the true relation is nonlinear in the case of U.S. economy. Some literature has found evidence

\footnotetext{
${ }^{8}$ The Bayesian analysis is based on 20,000 draws from the importance sampling density sescribed in Subsection $(2.3)$.
} 
of nonlinear effects of oil price on real economic activity of main industrialized countries from the studies of individual country analysis. Kilian and Vigfusson (2009), however, show that the regression models and estimation methods which use measures that censors energy price changes, produce inconsistent estimates of the true effects of unanticipated energy price increases and lead to overestimating the impact of energy price shocks on macroeconomy aggregates.

This paper examines the issue of whether the relation between oil price change and the business cycle is nonlinear along with two insights. First of all, this paper extends Hamilton's (2001) methodology for time-series data to the panel data framework to investigate whether the relation is nonlinear in terms of panel data analysis. Specifically, we consider nonlinear flexible inference with random error components. Secondly, our parametric approach does not have to use the censored oil price changes to avoid potential problems from using the censored energy prices changes.

Our results show from the study of the panel data for six industrialized countries that oil price increases are much more important than oil price decreases and previous upheaval in oil prices causes the marginal effect of any given oil price change to be reduced and support the claim in the literature. The alternative specifications of the panel data model with nonlinear flexible inference as a robustness analysis support similar nonlinear oil-macroeconomy relation. Therefore, the result of such a panel data analysis suggests that one should use a nonlinear function of oil price changes if the goal is to forecast GDP growth. 


\section{Appendix: Monte Carlo analysis for approximating the small- sample distribution of the LM test statistic}

In order to approximate the exact small-sample distribution of $\hat{\aleph}_{N T}$ and to examine how good the test statistic developed in equation (34) performs, we undertook a small-scale Monte Carlo analysis. We consider following linear data-generating process based on the linear error-components model:

$$
\begin{aligned}
y_{i t}= & 2.531-0.044 y_{i t-1}+0.068 y_{i t-2}+0.123 y_{i t-3}+0.068 y_{i t-4} \\
& +0.011 o_{i t-1}-0.005 o_{i t-2}-0.023 o_{i t-3}-0.039 o_{i t-4}+\varepsilon_{i t}, \\
\varepsilon_{i t}= & \omega_{i}+a_{t}+v_{i t},
\end{aligned}
$$

where $\omega_{i} \sim N(0,0.035), a_{t} \sim N(0,2.75), v_{i t} \sim N(0,21.39)$ and the disturbances are assumed to be mutually and temporally independent normal variables with variance equal to those estimated in the linear error-component model. Using 1,000 simulations of the data generated for this system corresponding to 6 countries oil price changes and GDP growth, we calculated the test statistic $\widehat{\aleph}_{N T}$ conditional on the best quadratic unbiased estimators for each simulation. We did

not find any case where $\hat{\aleph}_{N T}$ is greater than that of the original data, implying that the original data shows strong evidence on the nonlinear relation between oil price change and GDP growth. Furthermore, at the nominal $5 \%$ level of significance, the rejection rate of the linearity null hypothesis was $1.6 \%$, implying that the developed test statistic leads to some under-rejection of the true linearity null hypothesis but overall performance of the test statistic has good smallsample property in terms of conventional analysis. 


\section{Acknowledgments}

This work is supported by the Korea Research Foundation Grant funded by the Korean Government (MOEHRD) (KRF-2007-332-B00059). Deep discussions with James D. Hamilton are gratefully acknowledged. The author would like to thank the co-editor, Keith Blackburn, Ana Herrera, Chang Jin Kim, Soyoung Kim, Sun Bin Kim, Denise Osborn, Kwanho Shin, Donggyu Sul, Taku Yamamoto, two anonymous referees, and seminar participants at Cheonnam National University, Korea Institute of Finance, Korea University, University of Manchester, and 2007 Hitotsubashi Econometrics Conference, 2008 SETA Conference, 2008 Korea and the World Economy VII Conference, and 2008 ESEM Conference for helpful comments and suggestions and Wun Ho Lee for research assistance. The Korea University research grant and the financial support by the Institute of Economic Research at Korea University are also acknowledged. 


\section{References}

Amemiya T (1971) The estimation of the variance in a variance-components model. Int Econ Rev 12:1-13

Baltagi BH (2008) Econometric Analysis of Panel Data. John Wiley \& Sons LTD, Chinchester UK

Baltagi BH (1981) Pooling: An experimental study of alternative testing and estimation procedures in a two-way error component model. J Econom 17:21-49

Baltagi BH, Griffin JM (1983) Gasoline demand in the OECD: An application of pooling and testing procedures. Eur Econ Rev 22:117-137

Baltagi BH, Pinnoi N (1995) Public capital stock and state productivity growth: Further evidence from an error components model. Empir Econ 20:351-359

Baltagi BH, Song SH, Jung BC (2002) A comparative study of alternative estimators for the unbalanced two-way error component regression model. Econom J 5:480-493

Barsky RB, Kilian L (2002) Do We Really Know that Oil Caused the Great Stagflation? A Monetary Alternative. In: Bernanke BS, Rogoff K (ed) NBER Macroeconomics Annual 2001, MIT Press, Cambridge, USA, pp 137-183

Barsky RB, Kilian L (2004) Oil and the Macroeconomy Since the 1970s. J Econ Perspect 18(4):115-134

Blanchard OJ, Gali J (2008) The Macroeconomic Effects of Oil Price Shocks: Why are the 2000s so different from the 1970s?. Gali J, Gertler MJ (ed) International Dimensions of Monetary Policy, University of Chicago Press

Boehmer E, Megginson WL (1990) Determinants of secondary market prices for developing country syndicated loans. J Financ 45:1517-1540

Breusch TS (1987) Maximum Likelihood Estimation of Random Effects Models. J Econom $36: 383-389$

Burbidge J, Harrison A (1984) Testing for the Effects of Oil-Price Rises Using Vector Autoregressions. Int Econ Rev 25:459-484 
Carruth AA, Hooker MA, Oswald AJ (1998) Unemployment Equilibria and Input Prices: Theory and Evidence from the United States. Rev Econ Stat 80:621-628

Cuñado J, de Gracia FP (2003) Do oil price shocks matter? Evidence for some European countries. Energ Econ 25:137-154

Davis SJ (1987a) Fluctuations in the Pace of Labor Reallocation. In: Brunner K, Meltzer AH (ed) Empirical Studies of Velocity, Real Exchange Rates, Unemployment and Productivity. Carnegie-Rochester Conference Series on Public Policy 24, North Holland, Amsterdam, Netherlands

Davis SJ (1987b) Allocative Disturbances and Specific Capital in Real Business Cycle Theories. Am Econ Rev Pap Proc 77(2):326-332

Davis SJ, Haltiwanger J (2001) Sectoral Job Creation and Destruction Responses to Oil Price Changes. J Monet Econ 48:465-512

Dotsey M, Reid M (1992) Oil Shocks, Monetary Policy, and Economic Activity. Econ Rev of the Fed Reserve Bank of Richmond 78:14-27

Fuller WA, Battese GE (1973) Transformations for Estimation of linear models with nested error structure. J Am Stat Assoc 68:626-632

Geweke J (1989) Bayesian Inference in Econometrics Models Using Monte Carlo Integration. Econom 57:1317-1339

Gisser M, Goodwin TH (1986) Crude Oil and the Macroeconomy: Tests of Some Popular Notions. J Money Credit Banking 18:95-103

Hamilton JD (1983) Oil and the Macroeconomy Since World War II. J Political Econ 91:228-248 Hamilton JD (1996) This is What Happened to the Oil Price-Macroeconomy Relationship. J Monet Econ 91:228-248

Hamilton JD (2001) A Parametric Approach to Flexible Nonlinear Inference. Econom 69(3):537573

Hamilton JD (2003) What is an oil shock?. J Econom 113:363-398

Hamilton JD (2008) Oil and the Macroeconomy. Durlauf S., Blume L. (ed) New Palgrave Dictionary of Economics, Palgrave McMillan Ltd. 
Hamilton JD (2009a) Causes and Consequences of the Oil Shock of 2007-08. Brook Pap on Econ Act 1: 215-261

Hamilton JD (2009b) Yes, the Response of the U.S. Economy to Energy Prices is Nonlinear. http://dss.ucsd.edu/ jhamilto/Kilian_AER_comment.pdf

Hamilton JD, Herrera AM (2004) Oil Shocks and Aggregate Macroeconomic Behavior: The Role of Monetary Policy J Money Credit Banking 36(2):265-286

Hooker MA (1996) What Happened to the Oil Price-Macroeconomy Relationship?. J Monet Econ 38:195-213

Hsiao C (2003) Analysis of Panel Data. Cambridge University Press, Cambridge, UK

Jimenez-Rodriguez R, Sanchez M (2005) Oil Price Shocks and Real GDP growth: Empirical Evidence for Some OECD Countries. App Econ 37(2):201-228

Kilian L (2008a) Exogenous Oil Supply Shocks: How Big Are They and How Much Do They Matter for the U.S. Economy? Rev Econ Stat 90(2):216-240

Kilian L (2008b) A Comparison of the Effects of Exogenous Oil Supply Shocks on Output and Inflation in the G7 Countries. J Europ Econ Assoc 6(1):78-121

Kilian L (2009a) Not All Oil Price Shocks Are Alike: Disentangling Demand and Supply Shocks in the Crude Oil Market. Am Econ Rev 99(3): 1053-1069

Kilian L (2009b) Comment on "Causes and Consequences of the Oil Shock of 2007-08 by James D. Hamilton. Brook Pap on Econ Act 1: 267-278

Kilian L, Vigfusson RJ (2009) Pitfalls in Estimating Asymmetric Effects of Energy Price Shocks. http://www-personal.umich.edu/ lkilian/kv14apr09.pdf

Kim DH, Osborn DR, Sensier M (2005) Nonlinearity in the Fed's Monetary Policy Rule. J App Econom 20:621-639

Klevmarken NA (1989) Panel Studies: What Can We Learn From Them?. Europ Econ Rev $33: 523-529$

Leduc S, Sill K (2004) A Quantitative Analysis of Oil-Price Shocks, Systematic Monetary Policy and Economic Downturns. J Monet Econ 51:781-808 
Lee K, Ni S (2002) On the Dynamic Effects of Oil Price Shocks: A Study Using Industry Level Data. J Monet Econ 49:823-852

Lee K, Ni S, Ratti RA (1995) Oil Shocks and the Macroeconomy: The Role of Price Variability. Energ J 16:39-56

Loungani P (1986) Oil Price Shocks and the Dispersion Hypothesis. Rev Econ Stat 58:536-539

Maddala GS (1971) The Use of Variance Components Models in Pooling cross section and time series data. Econom 39(2):341-358

Mork KA (1989) Oil and the Macroeconomy When Prices Go Up and Down: An Extension of Hamilton's Results. J Political Econ 91:740-744

Mork KA, Olsen O, Mysen HT (1994) Macroeconomic Responses to Oil Price Increases and Decreases in Seven OECD Countries. Energy J 15(4):19-35

Nerlove ML (1971) A note on error components models. Econom 39:383-396

Rasche RH, Tatom JA (1977) Energy Resources and Potential GNP. Federal Reserve Bank of St. Louis Rev 59:10-24

Rasche RH, Tatom JA (1981) Energy Price Shocks, Aggregate Supply, and Monetary Policy: The Theory and International Evidence. In: Supply Shocks, Incentives, and National Wealth. Brunner K, Meltzer AH (ed) Carnegie-Rochester Conf Ser on Public Policy 14, North-Holland, Amsterdam, Netherlands:9-93

Rotemberg JJ, Woodford M (1996) Imperfect Competition and the Effects of Energy Price Increases. J Money Credit Banking 28:549-577

Swamy PAVB, Arora SS (1972) The exact finite sample properties of the estimators of coefficients in the error components regression models. Econom 40:261-275

Wallace TD, Hussain A (1969) The use of error components models in combining cross-section and time-series data. Econom 37:55-72 
Figure 1 Effect of oil prices on GDP growth one quarter later

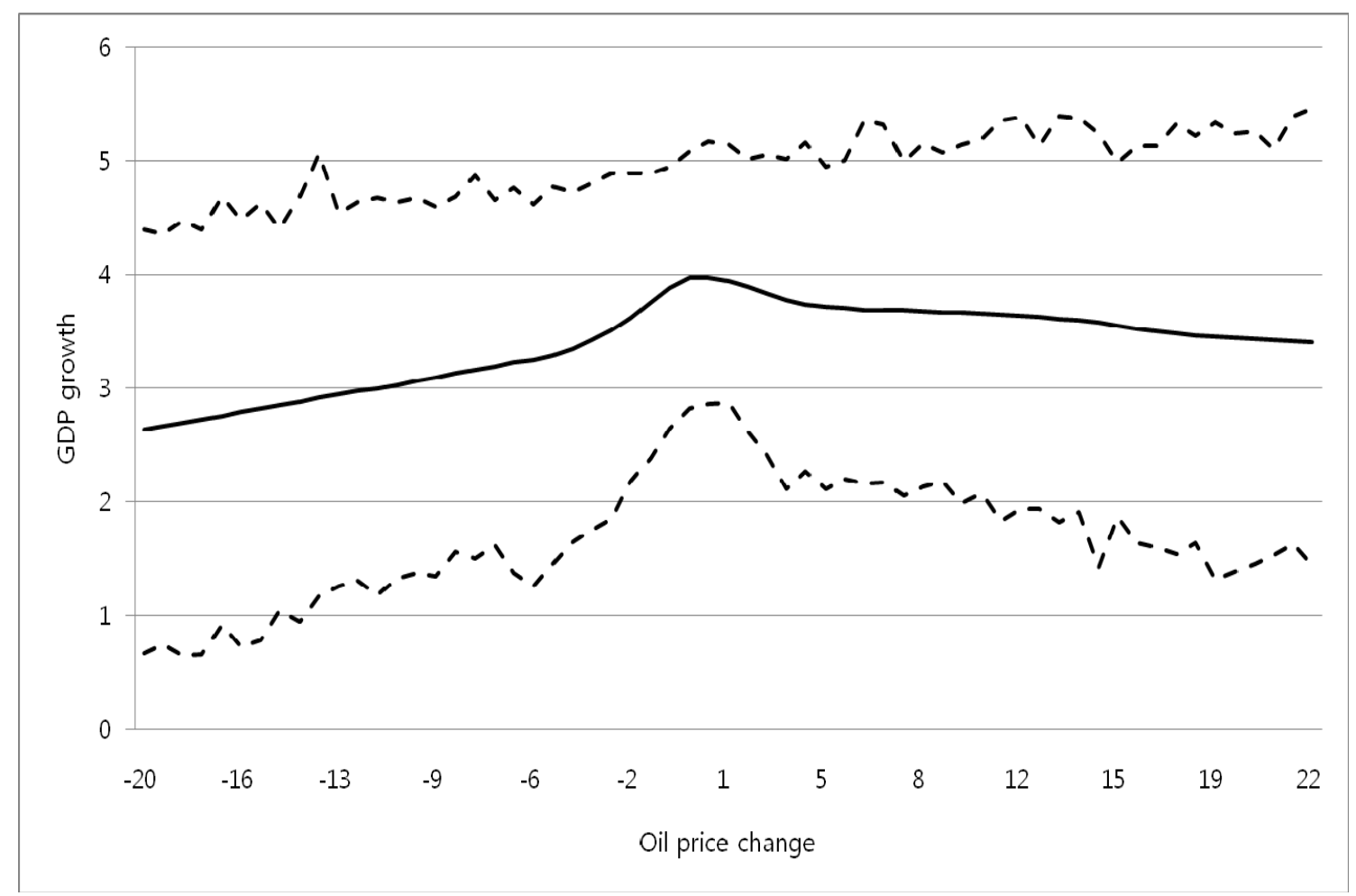

Solid line plots the posterior expectation of the function $\alpha_{0}+\boldsymbol{\alpha}^{\prime} \mathbf{x}_{i t}+\boldsymbol{\delta}^{\prime} \mathbf{z}_{i t}+\lambda m\left(\mathbf{x}_{i t}\right)$ evaluated at $\mathbf{x}_{i t}=\left(x_{i t-1}, \bar{o}_{i t-2}, \bar{o}_{i t-3}, \bar{o}_{i t-4}\right)^{\prime}$ and $\mathbf{z}_{i t}=\left(\bar{y}_{i t-1}, \bar{y}_{i t-2}, \bar{y}_{i t-3}, \bar{y}_{i t-4}\right)^{\prime}$ as a function of $x_{i t-1}$ where $\bar{z}_{i t-j}=T^{-1} \sum_{t=1}^{T} z_{i t-j}$ and where the expectation is with respect to the posterior distribution of $\quad \alpha_{0}, \boldsymbol{\alpha}, \delta, \lambda$, and $m\left(\mathbf{x}_{i t}\right) \quad$ conditional on observation of $\left\{y_{i t}, \mathbf{x}_{i t}, \mathbf{z}_{i t}\right\}$, for $t=1, \ldots, T ; i=1, \ldots, N$, with this posterior distribution estimated by Monte Carlo importance sampling with 100,000 simulations. Dashed lines give 95\% probability regions. 
Figure 2 Effect of oil prices on GDP growth two quarters later

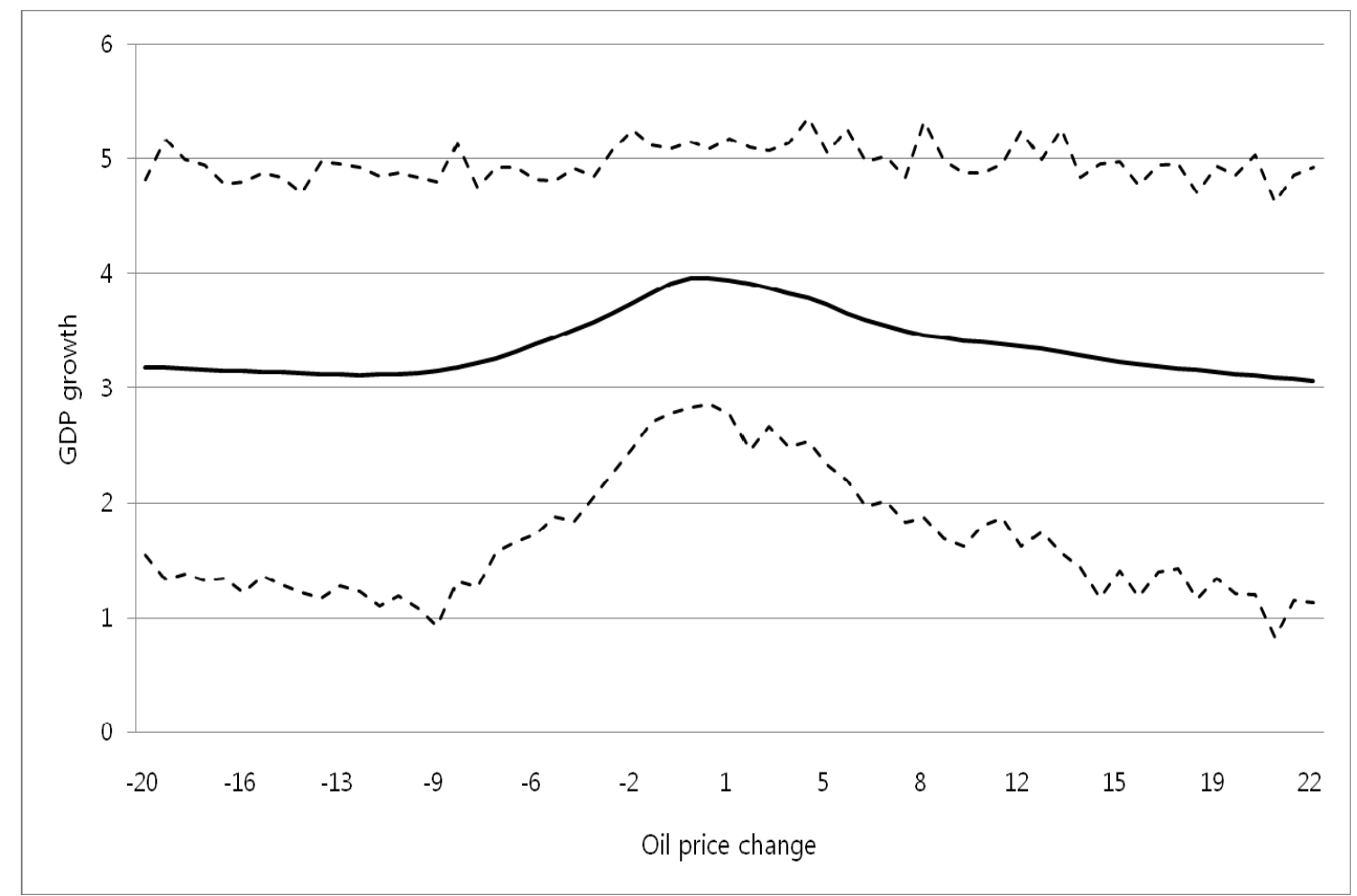

Solid line plots the posterior expectation of the function $\alpha_{0}+\boldsymbol{\alpha}^{\prime} \mathbf{x}_{i t}+\boldsymbol{\delta}^{\prime} \mathbf{z}_{i t}+\lambda m\left(\mathbf{x}_{i t}\right)$ evaluated at $\mathbf{x}_{i t}=\left(\bar{o}_{i t-1}, x_{i t-2}, \bar{o}_{i t-3}, \bar{o}_{i t-4}\right)^{\prime}$ and $\mathbf{z}_{i t}=\left(\bar{y}_{i t-1}, \bar{y}_{i t-2}, \bar{y}_{i t-3}, \bar{y}_{i t-4}\right)^{\prime}$ as a function of $x_{i t-2}$. 
Figure 3 Effect of oil prices on GDP growth three quarters later

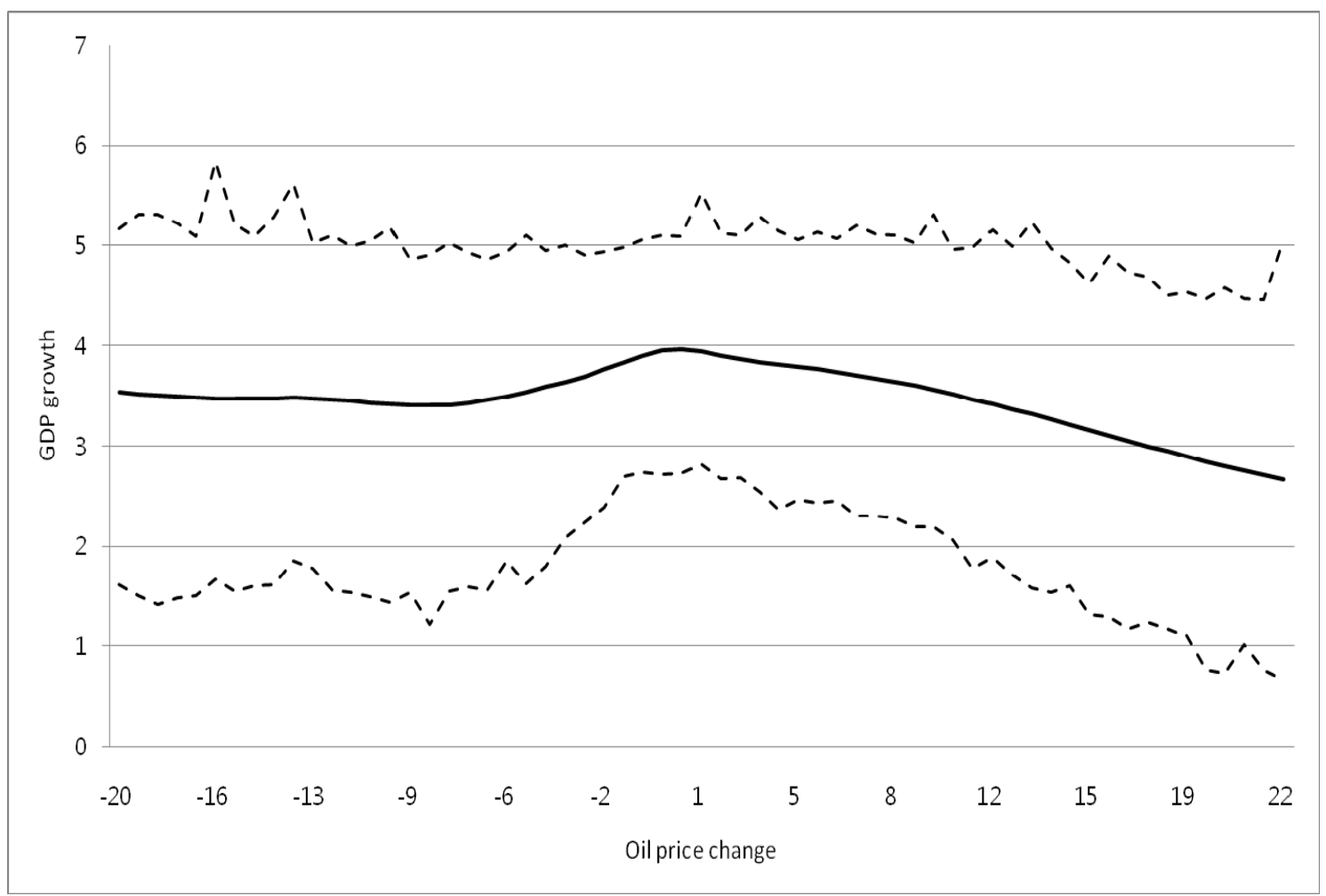

Solid line plots the posterior expectation of the function $\alpha_{0}+\boldsymbol{\alpha}^{\prime} \mathbf{x}_{i t}+\boldsymbol{\delta}^{\prime} \mathbf{z}_{i t}+\lambda m\left(\mathbf{x}_{i t}\right)$ evaluated at $\mathbf{x}_{i t}=\left(\bar{o}_{i t-1}, \bar{o}_{i t-2}, x_{i t-3}, \bar{o}_{i t-4}\right)^{\prime}$ and $\mathbf{z}_{i t}=\left(\bar{y}_{i t-1}, \bar{y}_{i t-2}, \bar{y}_{i t-3}, \bar{y}_{i t-4}\right)^{\prime}$ as a function of $x_{i t-3}$. 
Figure 4 Effect of oil prices on GDP growth four quarters later

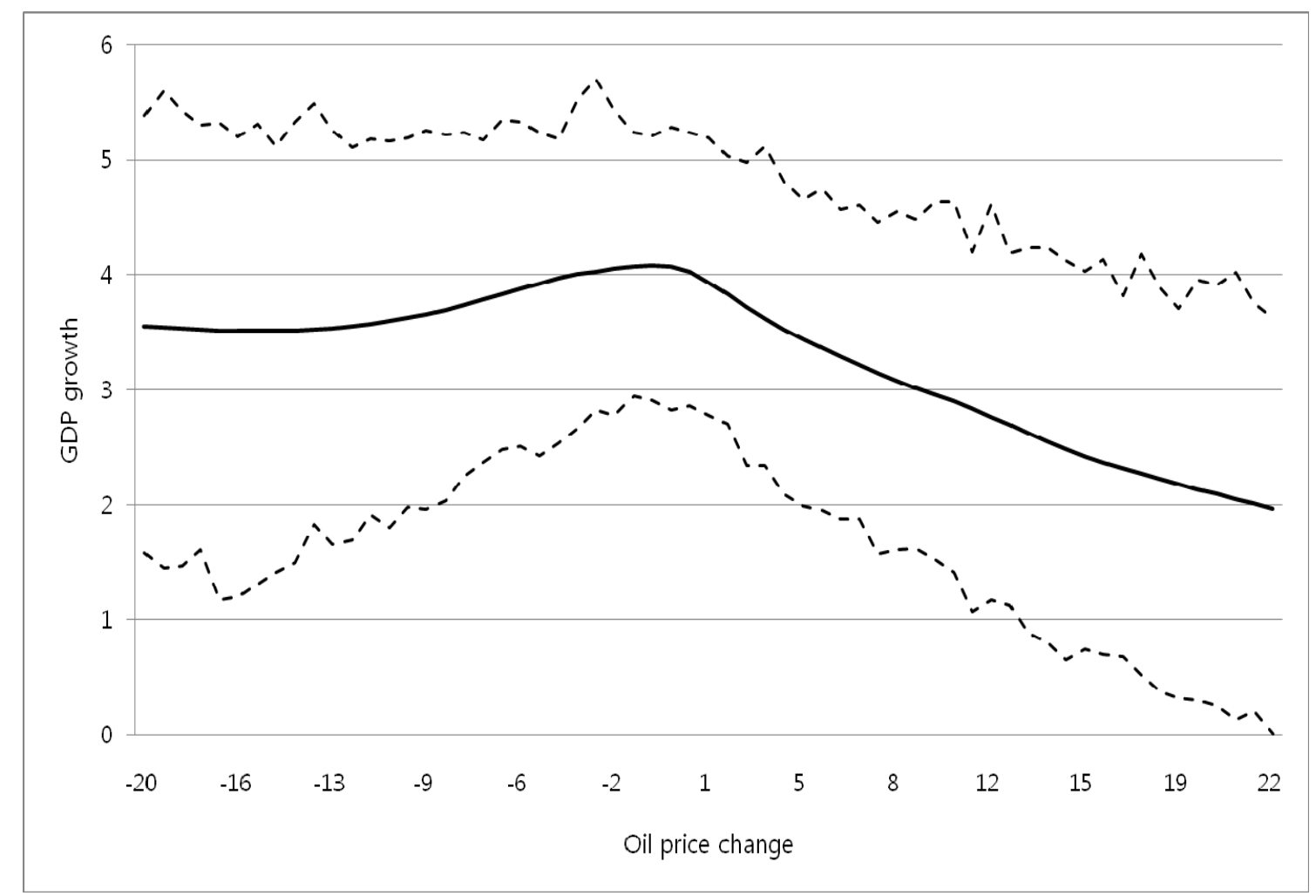

Solid line plots the posterior expectation of the function $\alpha_{0}+\boldsymbol{\alpha}^{\prime} \mathbf{x}_{i t}+\boldsymbol{\delta}^{\prime} \mathbf{z}_{i t}+\lambda m\left(\mathbf{x}_{i t}\right)$ evaluated at $\mathbf{x}_{i t}=\left(\bar{o}_{i t-1}, \bar{o}_{i t-2}, \bar{o}_{i t-3}, x_{i t-4}\right)^{\prime}$ and $\mathbf{z}_{i t}=\left(\bar{y}_{i t-1}, \bar{y}_{i t-2}, \bar{y}_{i t-3}, \bar{y}_{i t-4}\right)^{\prime}$ as a function of $x_{i t-4}$. 
Figure 5 Effect of oil prices on GDP growth three quarters later different possible values of $o_{t-4}$

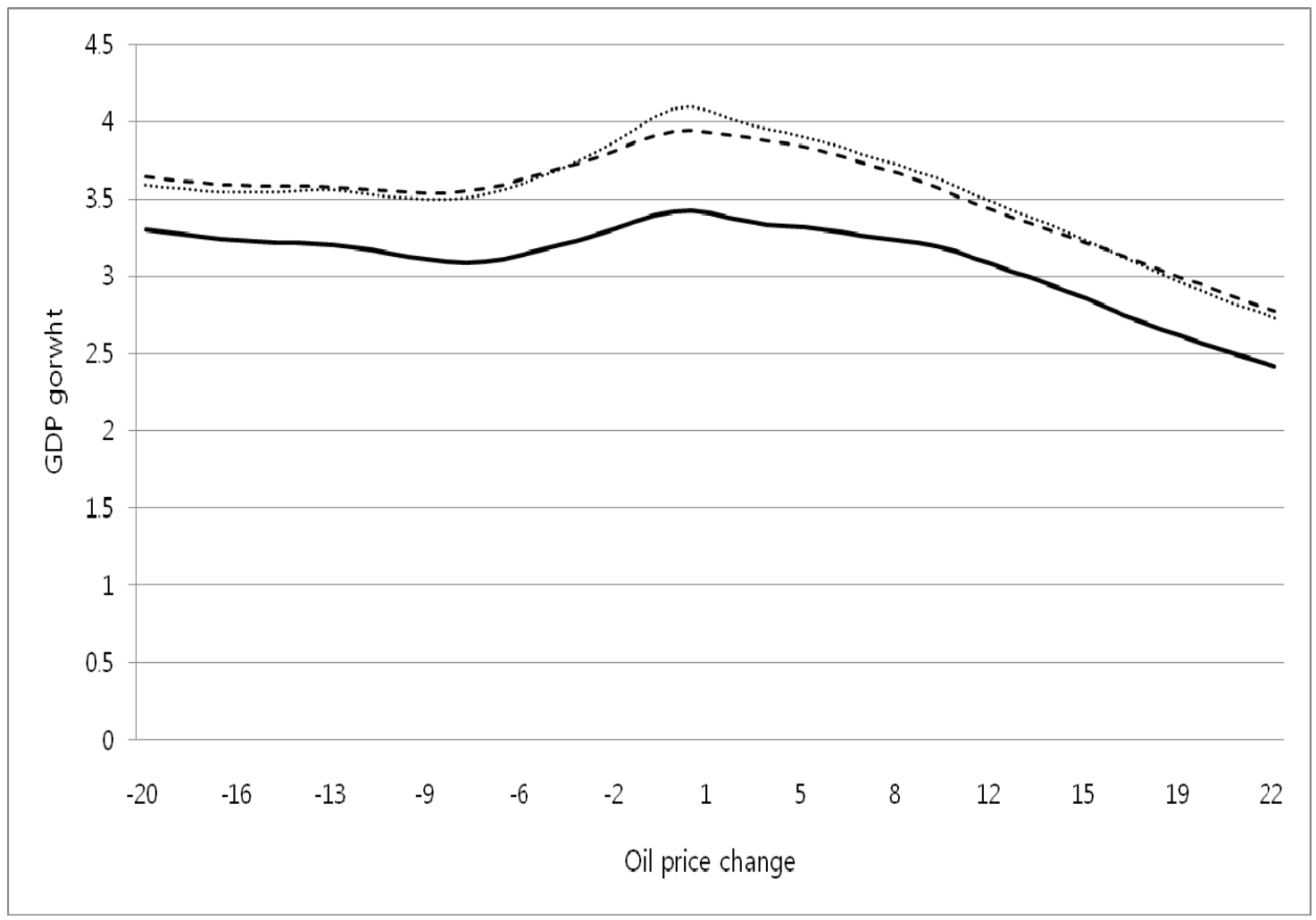

Three lines plot the posterior expectation of the function $\alpha_{0}+\boldsymbol{\alpha}^{\prime} \mathbf{x}_{i t}+\boldsymbol{\delta}^{\prime} \mathbf{z}_{i t}+\lambda m\left(\mathbf{x}_{i t}\right)$ evaluated at $\mathbf{x}_{i t}=\left(\bar{o}_{i t-1}, \bar{o}_{i t-2}, x_{i t-3}, x_{i t-4}\right)^{\prime}$ and $\mathbf{z}_{i t}=\left(\bar{y}_{i t-1}, \bar{y}_{i t-2}, \bar{y}_{i t-3}, \bar{y}_{i t-4}\right)^{\prime}$ as a function of $x_{i t-3}$. For the solid line, $x_{i t-4}=5$, for the dashed line, $x_{i t-4}=-5$, and for the dotted line, $x_{i t-4}=0$. 
Figure 6a Effect of oil prices on GDP growth four quarters later: With fixed effect

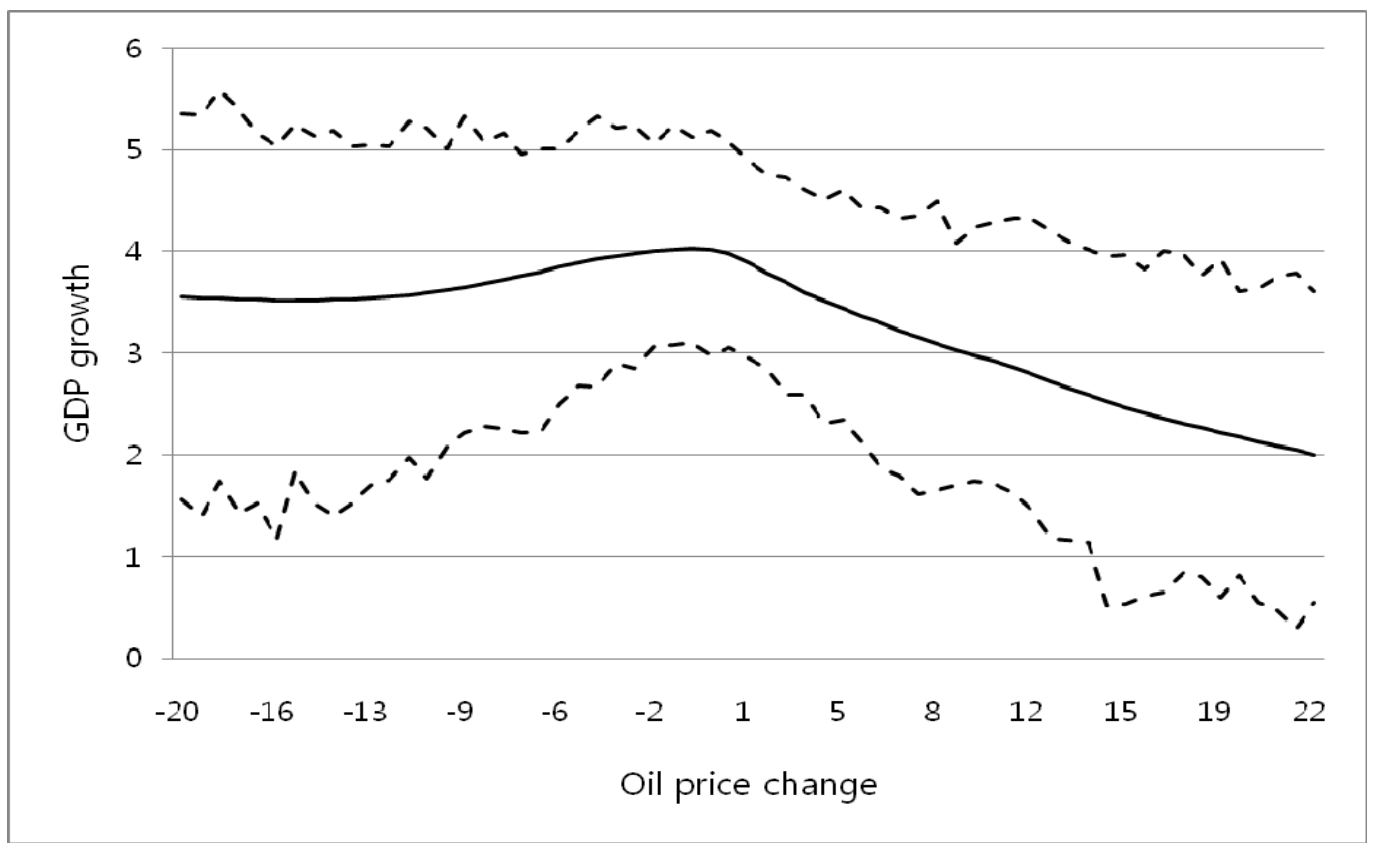

Figure 6b Effect of oil prices on GDP growth four quarters later: Without country specific effect

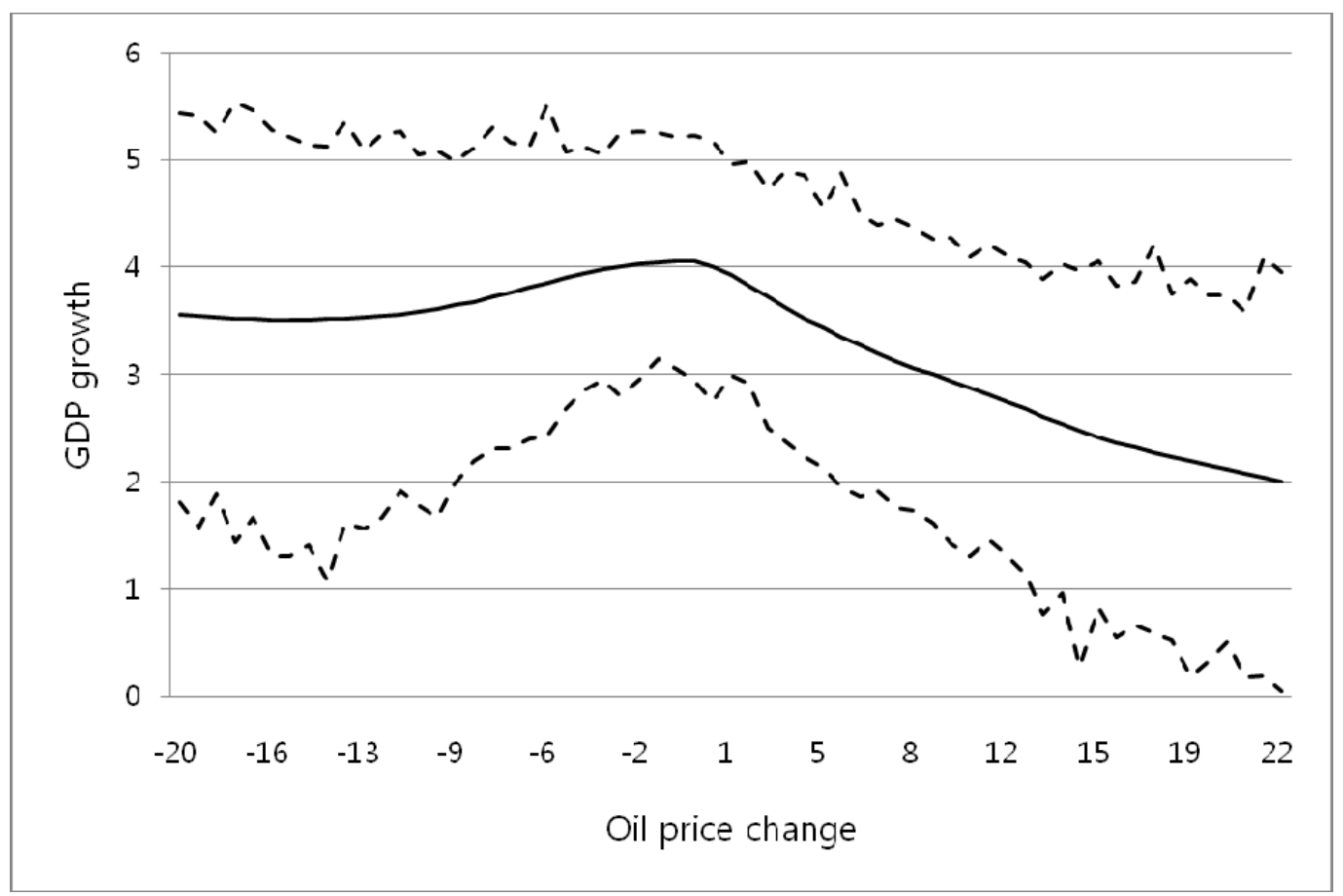


Figure 7a Effect of oil prices on GDP growth three quarters later different possible values of $o_{t-4}$ : With fixed effect

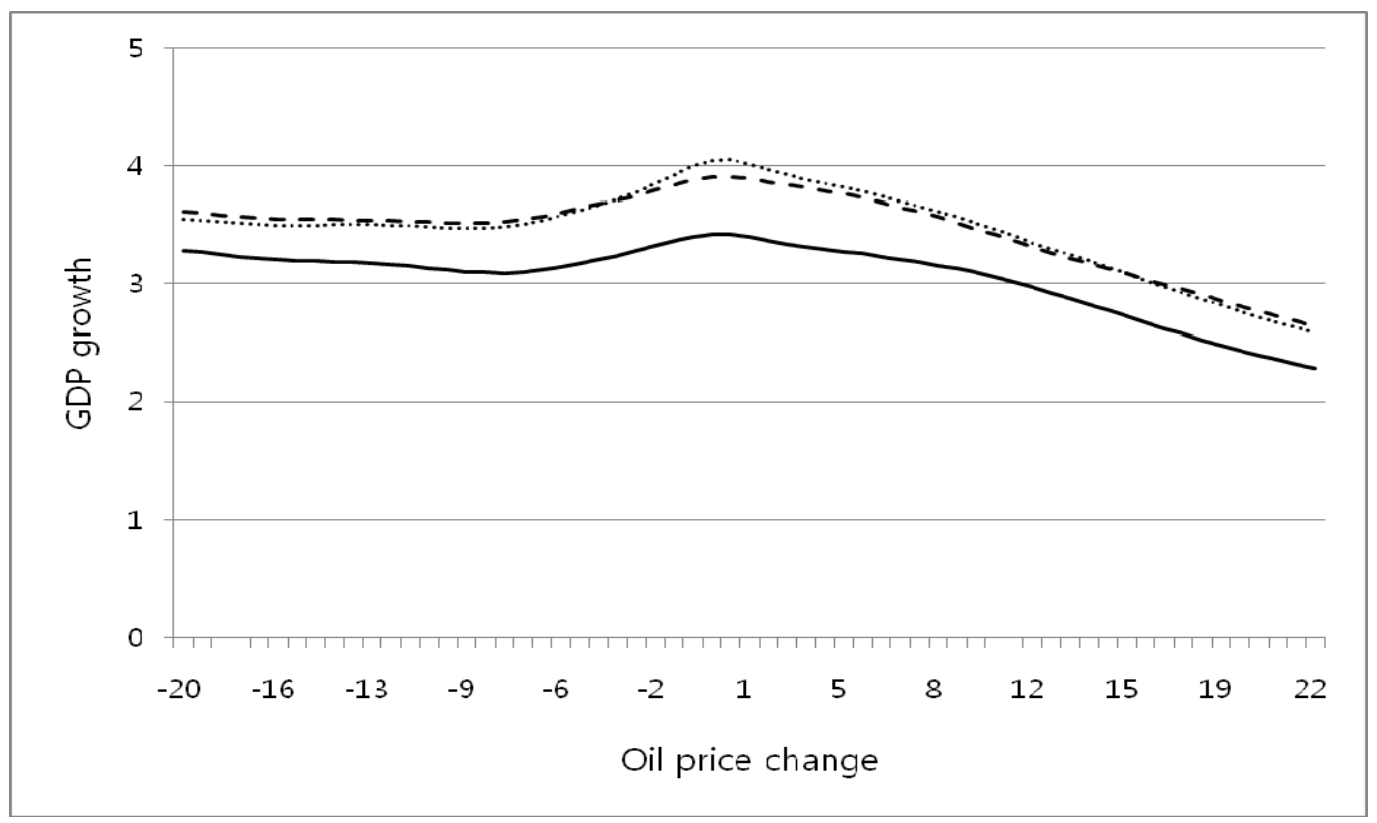

Figure 7b Effect of oil prices on GDP growth three quarters later different possible values of $o_{t-4}$ : Without country specific effect

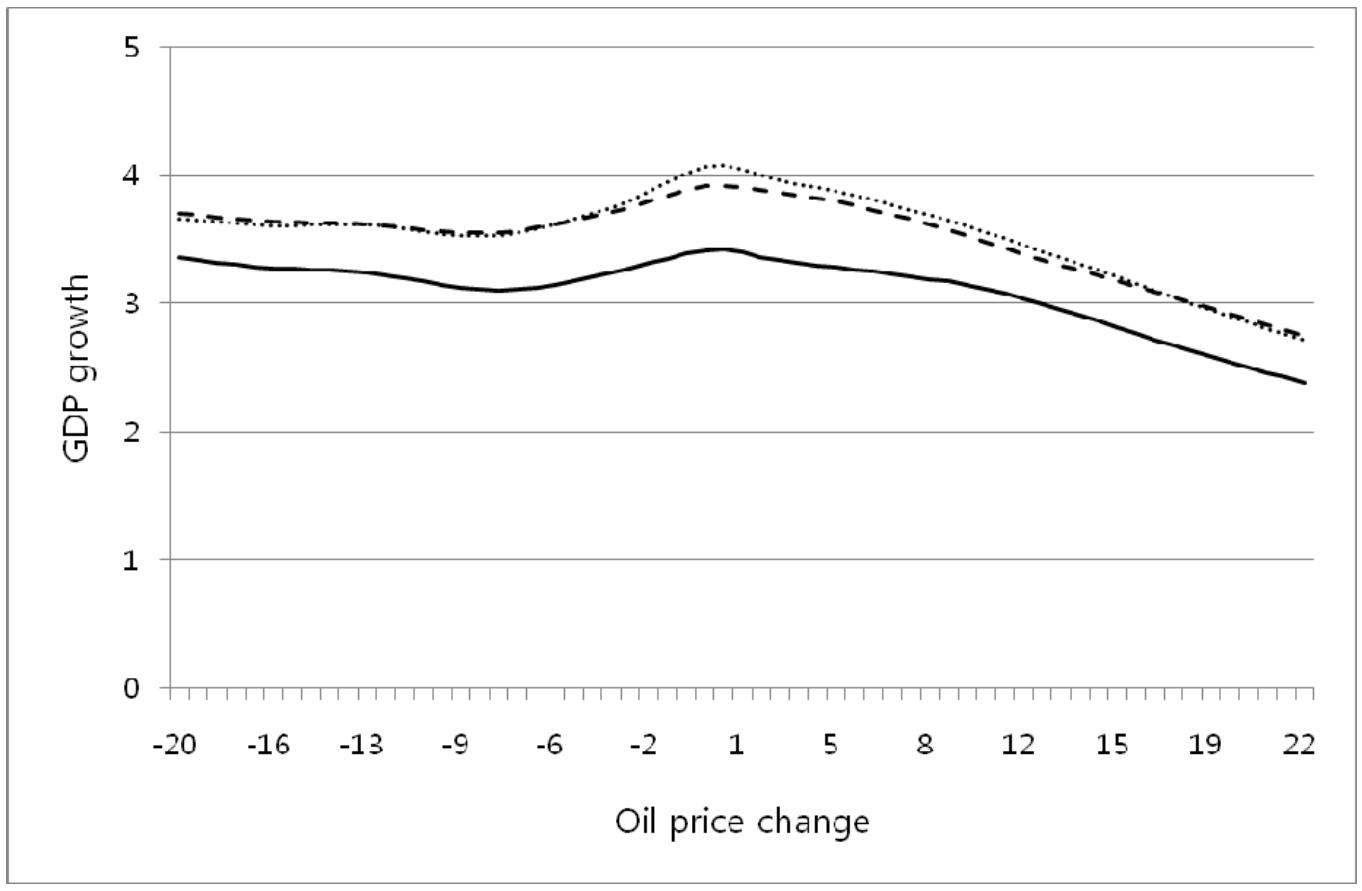

